\title{
Redox-responsive hyaluronic acid-functionalized graphene oxide nanosheets for targeted delivery of water-insoluble cancer drugs
}

This article was published in the following Dove Press journal: International Journal of Nanomedicine

\author{
Jian Liu ${ }^{1,2}$ \\ Doudou Zhang ${ }^{1,2}$ \\ Shu Lian ${ }^{1,2}$ \\ Junxia Zheng ${ }^{1,2}$ \\ Bifei $\mathrm{Li}^{1,2}$ \\ Tao $\mathrm{Li}^{1,2}$ \\ Lee Jia ${ }^{1,2}$ \\ 'Cancer Metastasis Alert \\ and Prevention Center, and \\ Biopharmaceutical Photocatalysis, \\ State Key Laboratory of \\ Photocatalysis on Energy and \\ Environment, Fuzhou University, \\ Fuzhou 350002, China; ${ }^{2}$ Fujian \\ Provincial Key Laboratory of Cancer \\ Metastasis Chemoprevention and \\ Chemotherapy, Fuzhou University, \\ Fuzhou 350002, China
}

Background: Gefitinib (Gef), an important epidermal growth factor receptor (EGFR), is used to treat lung cancer, but low water solubility and poor bioavailability severely limit its application in cancer therapy.

Methods: In this study, nano-graphene oxide (NGO) was decorated with hyaluronic acid (HA) by a linker cystamine dihydrochloride containing disulfide bonds (-SS-), followed by the incorporation of gefitinib, thus, constructing a HA-functionalized GO-based gefitinib delivery system (NGO-SS-HA-Gef). Subsequently, studies of biological experiments in vitro and in vivo were performed to investigate the therapeutic effect of the system in lung cancer.

Results: The HA-grafted GO nanosheets possessed enhanced physiological stability, admirable biocompatibility, and no obvious side effects in mice and could act as a nanocarrier for the delivery of gefitinib to tumor. Cellular uptake and intracellular cargo release assays showed that the uptake of NGO-SS-HA by A549 cells was facilitated via CD44 receptor-mediated endocytosis, and that more drug was released from NGO-SS-HA in the presence of GSH than in the absence of GSH. The target-specific binding of NGO-SS-HA to cancer cells with redox-responsive cargo release significantly enhanced the abilities of gefitinib-loaded GO nanosheets to induce cell apoptosis, suppress cell proliferation, and inhibit tumor growth in lung cancer cell-bearing mice.

Conclusion: The results demonstrated the potential utility of NGO-SS-HA-Gef for therapeutic applications in the treatment of lung cancer.

Keywords: nano-graphene oxide, gefitinib, hyaluronic acid, CD44, redox-responsive

\section{Introduction}

Lung cancer, one of the most common cancers in the world, has become the leading cause of cancer-related death globally, with non-small-cell lung cancer accounting for $\sim 80 \% .^{1-3}$ Hence, increasing attention has been paid to the study of drugs against lung cancer. In the past few decades, researchers have developed many anti-lung cancer drugs to extend patient survival time, such as paclitaxel, ${ }^{4,5}$ docetaxel, ${ }^{6,7}$ and cisplatin. ${ }^{8,9}$ In addition, a few drugs specifically targeted against lung cancer have been used in clinical treatment, such as gefitinib (Gef). ${ }^{10-12}$ Gef can restrict the activity of epidermal growth factor receptor tyrosine kinase (EGFR-TK), enhance the apoptosis of tumor cells, and then inhibit tumor growth. Although Gef has shown strong potential in the treatment of lung cancer, it is limited in this application by some of its clear defects, ${ }^{13-15}$ such as low water solubility, poor bioavailability, and side effects. Therefore, developing a Gef delivery system is necessary to overcome these shortcomings and improve its bioavailability.

Graphene oxide (GO), a prominent type of two-dimensional material, has attracted tremendous attention in many fields, including biomedicine, in recent years because
Correspondence: Lee Jia

Fujian Provincial Key Laboratory of Cancer Metastasis Chemoprevention and Chemotherapy, Fuzhou University, 2 Xueyuan Road, Sunshine Technology Building, 6FL, Fuzhou University, Fuzhou, Fujian 350116, China

$\mathrm{Tel}+86$ I5I 5963020 I

Email cmapcjia1234@163.com

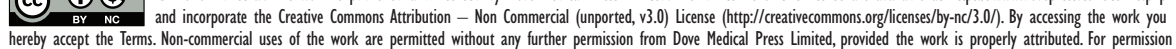
for commercial use of this work, please see paragraphs 4.2 and 5 of our Terms (https://www.dovepress.com/terms.php). 
of its unique properties. ${ }^{16-20}$ However, GO aggregates rapidly in solutions rich in salts or proteins, which hinders the application of GO in biomedicine. Sun et al first reported that nano-graphene oxide (NGO) decorated with polyethylene glycol (PEG) could be dispersed evenly in both physiological solution and cell medium and act as a nanocarrier for doxorubicin loading in intracellular imaging and drug delivery. ${ }^{21}$ Subsequently, Song et al presented hyaluronic acid (HA)decorated $\mathrm{GO}$ as a nanocarrier for targeted and $\mathrm{pH}$-responsive anticancer drug delivery. ${ }^{22} \mathrm{HA}$, a water-soluble mucopolysaccharide, has excellent biodegradability, biocompatibility, and non-immunogenicity. Moreover, it can also specifically bind the cluster determinant 44 (CD44) receptor, which is overexpressed on the surface of various tumor cells. ${ }^{23-25} \mathrm{HA}$ function not only improves the stability of GO in PBS and cell medium but also enhances the uptake of GO by CD44 receptor-overexpressing cells via the specific interaction between the CD44 receptor and HA.

However, similar to $\mathrm{PEG},{ }^{26} \mathrm{HA}$ forms a shell on the surface of nanosheets after combination with NGO, and this shell could restrict the release of a loaded drug from the nanocarrier, which seriously attenuates the therapeutic efficacy of the nanosheets. Therefore, it is necessary to utilize special means to remove the HA barrier for accelerating drug release, such as a redoxresponsive mechanism. The redox environment in a tumor cell is an important parameter that may determine the response of a tumor to certain chemotherapeutic agents and radiation. Various intracellular molecules may contribute to the overall redox status in tissues, including glutathione (GSH). ${ }^{26-29} \mathrm{GSH}$, a tripeptide consisting of glutamic acid, cysteine, and glycine, can be found in almost all cells in the body, and the intracellular concentration of GSH is significantly higher than its extracellular concentration. ${ }^{30,31}$ A triggering mechanism based on the evident difference in GSH concentration could therefore be utilized to remove the diffusion barrier caused by HA.

In the current work, a new NGO-based drug delivery system was developed and decorated with HA via disulfide bonds (Figure 1A). The resulting NGO-SS-HA-Gef can not only improve the above shortcomings of Gef in the treatment of lung cancer but also promote the accumulation of nanosheets in tumor sites and facilitate drug release from the nanosheets in response to tumor-relevant GSH. Briefly, HA was conjugated onto the surface of NGO with a linker, cystamine dihydrochloride. The successful preparation of NGO-SS-HA was confirmed by infrared (IR) spectroscopy, UV-vis spectroscopy, and atomic force microscopy (AFM). Then, Gef was physically loaded onto NGO-SS-HA via $\pi-\pi$ stacking and hydrophobic interactions to afford a nanosheet complex, NGO-SS-HA-Gef. The surface-engineered structure of NGO-SS-HA-Gef was found to accelerate the release of Gef from the nanosheets in the presence of GSH. Next, rhodamine $\mathrm{B}$ (RB), a fluorescent dye, was loaded onto the NGO-SS-HA to generate a nanohybrid, NGO-SS-HA-RB, which was used to investigate the cellular uptake of NGO-SS-HA and intracellular cargo release from the nanosheets. The in vitro cytotoxicity of NGO-SS-HA-Gef against cells was evaluated in a dose- and time-dependent manner. Finally, a series of in vivo studies were conducted to investigate the tissue biodistribution of NGO-SS-HA-Gef and evaluate its efficacy in tumor therapy.

\section{Experimental section Materials}

The native graphite flake was purchased from Alfa Aesar ( $\geq 99.8 \%$, Ward Hill, MA, USA). $\mathrm{NaCl}$ ( $\geq 99.0 \%$ ), $\mathrm{H}_{3} \mathrm{PO}_{4}$ ( $\geq 85.0 \%), \mathrm{H}_{2} \mathrm{SO}_{4}(98.0 \%), \mathrm{H}_{2} \mathrm{O}_{2}(\geq 30.0 \%)$, and $\mathrm{HCl}(\geq 36.0 \%)$ were acquired from Sinopharm Chemical Reagent Co. Ltd (Shanghai, China). Cystamine dihydrochloride and hexamethylenediamine (HMD) were purchased from Bide Pharmatech Ltd (Shanghai, China). Hyaluronic acid ( $M W=35 \mathrm{kDa}$ ) was obtained from Fureda Biological Technology Co., Ltd (Jinan, China). Gefitinib was purchased from Meilun Biological Technology Co., Ltd (Dalian, China). Cell Counting Kit-8 (CCK-8) and Ham's F-12K (Kaighn's) Medium were purchased from Dingguo Changsheng Biotechnology Co., Ltd (Beijing, China). Glutathione reduced ethyl ester (GSH-OEt, $\geq 90 \%$ ) and glutathione (98\%) were purchased from Sigma-Aldrich Co. (St Louis, MO, USA). All other reagents were purchased from J\&K Scientific Ltd (Shanghai, China) and used without further purification.

\section{Preparation of NGO}

$\mathrm{GO}$ was prepared from native graphite flake following an improved Hummer's method. ${ }^{32}$ The resulting GO was mixed with $\mathrm{NaOH}$ aqueous solution $(4 \mathrm{mg} / \mathrm{mL})$, and the mixed solution was sonicated at $1,000 \mathrm{~W}$ for 3 hours to convert $\mathrm{OH}$ groups to $\mathrm{COOH}$ groups. The resulting $\mathrm{GO}-\mathrm{COOH}$ solution was neutralized with dilute $\mathrm{HCl}$, dialyzed in water for 1 week to remove impurities, and filtered through a $0.45 \mu \mathrm{m}$ filter to fully remove any large-scale GO-COOH for NGO-COOH.

\section{Synthesis of NGO-SS-HA}

HA was dispersed in 2-(N-Morpholino) ethanesulfonic acid buffer ( $\mathrm{pH}$ 6.2) followed by the addition of hydrochloride (EDC) $(50 \mathrm{mg})$ and 1-hydroxybenzotriazole (70 mg). The mixed solution was stirred vigorously for 1 hour. The activated HA was added dropwise into $20 \mathrm{~mL}$ of cystamine dihydrochloride aqueous solution $(56 \mathrm{mg} / \mathrm{mL})$ over 10 minutes, and then the solution was stirred overnight. 
A

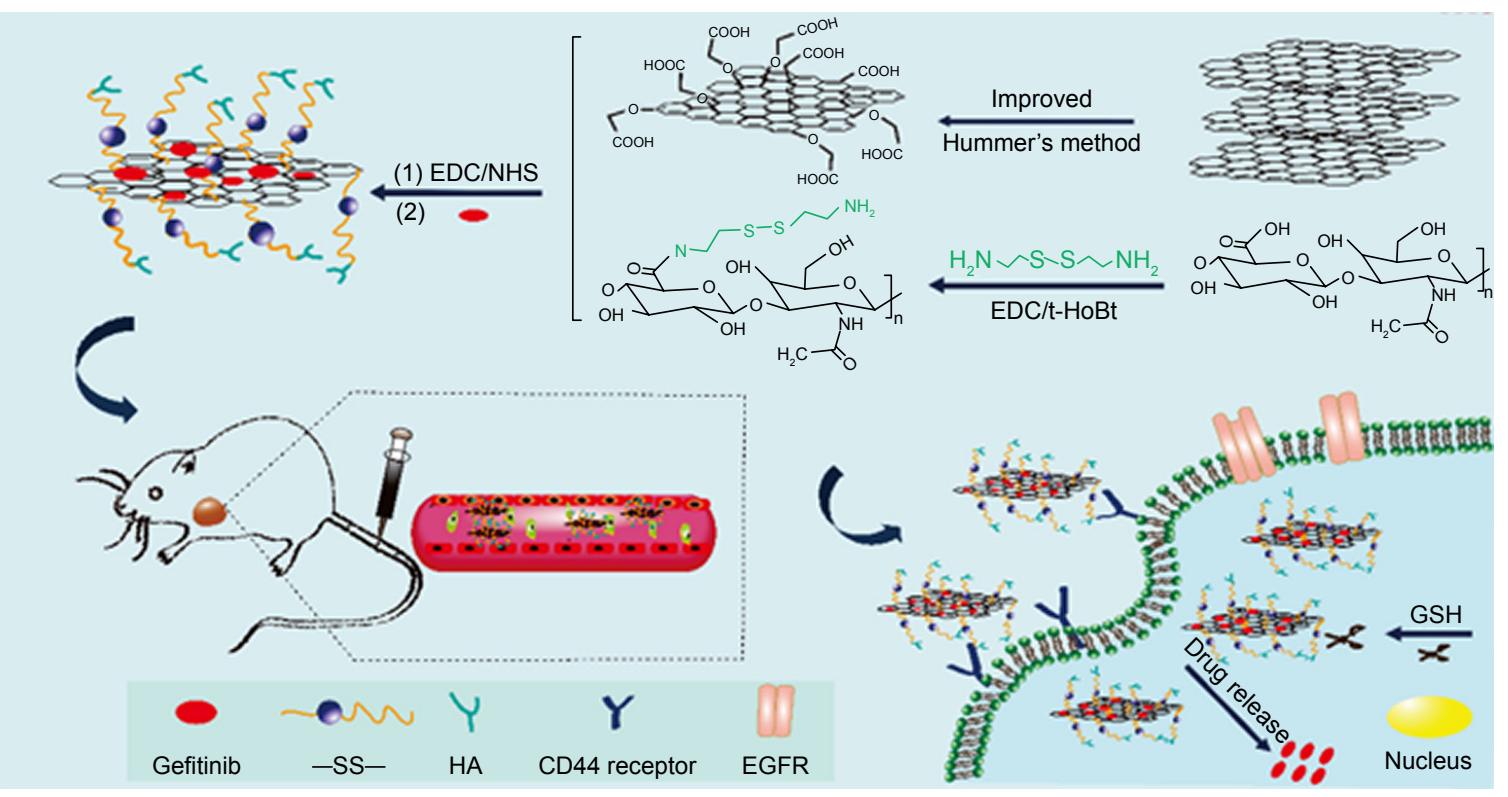

B

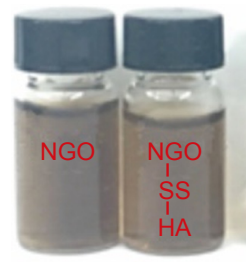

Water

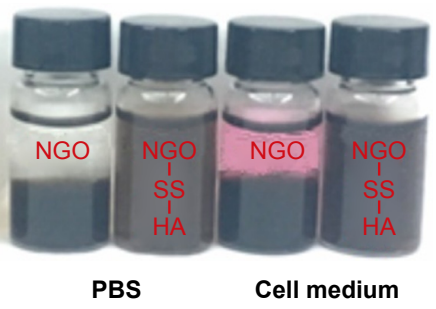

PBS
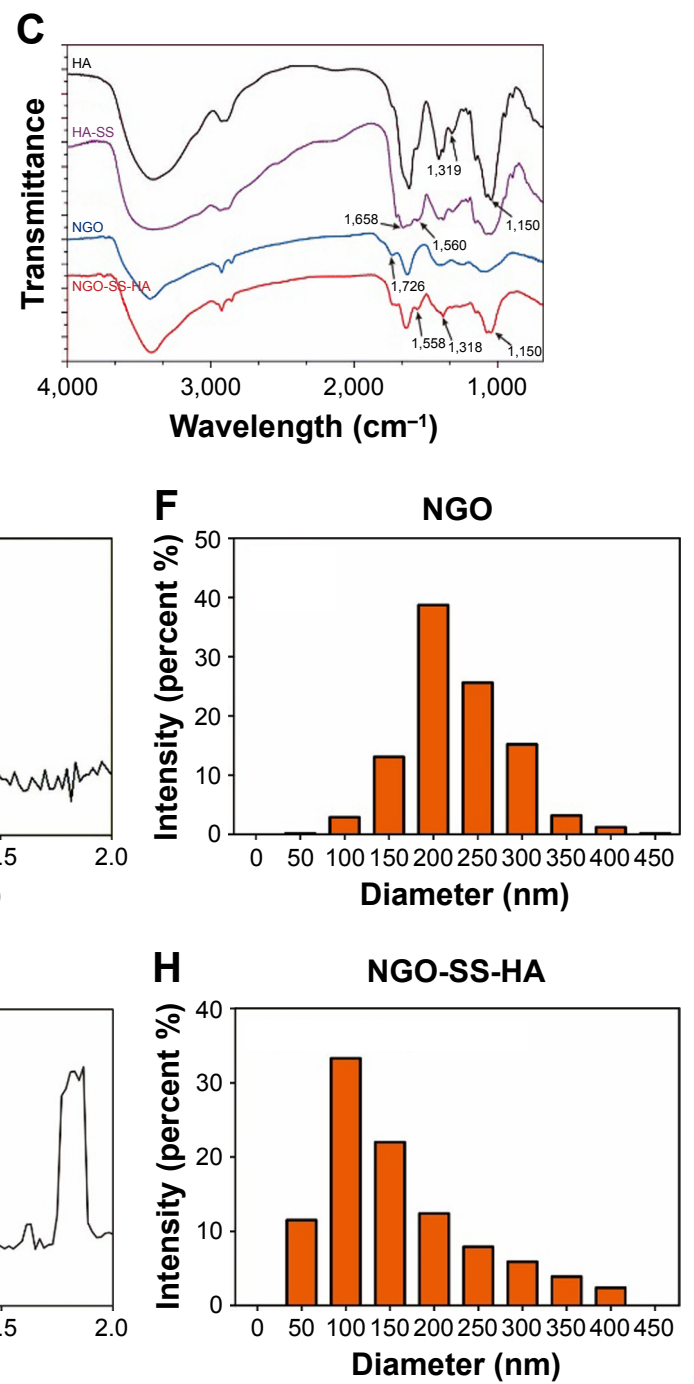

Figure I (A) Scheme showing the fabrication process of NGO-SS-HA-Gef, its capability to target cancer cells, and intracellular drug release triggered by redox responsiveness to GSH. (B) Images of NGO and NGO-SS-HA in water, PBS, and cell medium for I week. FTIR spectra (C) of HA, HA-SS, NGO, and NGO-SS-HA; AFM images (D) of NGO and NGO-SS-HA. (E and $\mathbf{G}$ ) The thickness of NGO and NGO-SS-HA measured by AFM. (F and $\mathbf{H})$ Histograms of NGO and NGO-SS-HA diameters, respectively. Abbreviations: NGO, nano-graphene oxide; HA, hyaluronic acid; Gef, gefitinib; GSH, glutathione; FTIR, Fourier transform infrared; AFM, atomic force microscopy. 
HA-SS-NH2 was acquired by freeze-drying after dialyzing against water for 2 days.

For the synthesis of NGO-SS-HA, $10 \mathrm{~mL}$ of $\mathrm{NGO}$ solution $(1 \mathrm{mg} / \mathrm{mL})$ was incubated with EDC $(15 \mathrm{mg})$ and n-hydroxysuccinimide (NHS, $18 \mathrm{mg}$ ) for 1 hour. The resulting $\mathrm{N}$-hydroxysuccinimide-activated NGO was washed with water several times by ultrafiltration to remove residual EDC and NHS, and then HA-SS-NH2 aqueous solution $(2 \mathrm{mg} / \mathrm{mL})$ was added. After sonication for 30 minutes and stirring for 24 hours, non-conjugated HA-SS-NH2 was removed by ultrafiltration. The resulting NGO-SS-HA was stored at $4^{\circ} \mathrm{C}$ until use.

\section{Loading of Gef on NGO-SS-HA}

NGO-SS-HA aqueous solution $(0.4 \mathrm{mg} / \mathrm{mL})$ was mixed with various concentrations of Gef in dimethyl sulfoxide (DMSO) solution and stirred for 24 hours. Excess Gef precipitated as solid was removed by centrifugation at 5,000 rpm for 20 minutes. The supernatant was filtered through a $0.45 \mu \mathrm{m}$ filter to fully remove any solids and then washed five times by ultrafiltration to remove the small amount of solubilized Gef. The obtained NGO-SS-HA-Gef was re-suspended in PBS and stored until use. The UV-vis spectra of NGO-SS-HA before and after drug loading were recorded to determine the drug loading ratio.

\section{GSH-induced Gef release in vitro}

To evaluate the $\mathrm{pH}$ and redox-responsive release property, NGO-SS-HA-Gef in PBS at different $\mathrm{pH}$ values (5.5 and 7.4) and GSH concentrations $(0 \mu \mathrm{M}, 2 \mu \mathrm{M}$ and $10 \mathrm{mM})$ was loaded into dialysis tubes. At designated time points, $0.2 \mathrm{~mL}$ of each dialysis solution was removed, and the same volume of fresh corresponding buffer was added. The amount of Gef released was measured by the UV-vis spectra at a wavelength of $343 \mathrm{~nm}$.

\section{Cell culture}

A549 cells and HELF cells were purchased from the Cell Resource Center of Shanghai Institute for Biological Sciences (Chinese Academy of Sciences). A549 cells were cultured in Ham's F12K medium (Sigma-Aldrich Co., Ltd.), and HELF cells were cultured in DMEM. The medium was supplemented with 10\% (v/v) fetal bovine serum (Invitrogen) and $1 \%$ penicillin/streptomycin. The cells were all maintained at $37^{\circ} \mathrm{C}$ in a humidified atmosphere with $5 \% \mathrm{CO}_{2}$.

\section{Cytotoxicity of NGO-SS-HA against cells in vitro}

The cytotoxicity of NGO-SS-HA against cells was measured using the CCK-8 assay. A549 and HELF cells were seeded into 96-well cell culture plates at $1 \times 10^{4} /$ well for 24 hours and incubated with NGO-SS-HA at various concentrations for another
24, 48, and 72 hours. Next, the absorbance of each well was measured at $450 \mathrm{~nm}$ with a microplate reader (Tecan, Männedorf, Switzerland). The cell viability was computed by the following equation: Cell viability $=\left(\mathrm{OD}_{\text {treated }} / \mathrm{OD}_{\text {control }}\right) \times 100 \%$.

\section{Hemolysis assays in vitro}

Blood samples (obtained from a healthy a mouse) were stabilized with ethylenediaminetetraacetic acid. Red blood cells were separated from serum by centrifugation at 1,000 rpm for 10 minutes, washed several times with PBS, and then diluted with PBS. Then, $0.3 \mathrm{~mL}$ of diluted suspension was mixed with 1) $0.9 \mathrm{~mL}$ of PBS as a negative control, 2) $0.9 \mathrm{~mL}$ of deionized water as a positive control, or 3) $0.9 \mathrm{~mL}$ of NGO-SS-HA dispersions at various concentrations. Next, all the mixtures were shaken at $100 \mathrm{rpm}$ for 2 hours at $37^{\circ} \mathrm{C}$ and centrifuged at $14,000 \mathrm{rpm}$. The absorbance of the supernatants at $541 \mathrm{~nm}$ was measured by UV-vis spectroscopy. Hemolysis percentage $(\%)=\left(\mathrm{A}_{\text {treated }}-\mathrm{A}_{\text {negative }}\right) /\left(\mathrm{A}_{\text {positive }}-\mathrm{A}_{\text {negative }}\right) \times 100 \%$.

\section{Cellular uptake of NGO-SS-HA}

To study the mechanism of the nanosheets entering into cells, $\mathrm{RB}$ was used as a fluorescent probe to determine the ability of the HA-modified GO nanosheets to specifically bind CD44 receptor-overexpressing cancer cells. The loading of RB onto NGO-SS-HA was similar to that of Gef.

A549 (CD44 receptor-positive) and HELF cells (CD44 receptor-negative) were seeded separately into confocal dishes at densities of $1 \times 10^{5}$ and $2 \times 10^{5}$ cell/dish and allowed to grow for 24 hours. Next, the cells were treated with 0 or $5 \mathrm{mg} / \mathrm{mL}$ HA for 2 hours and then incubated with NGO-SS-HA-RB ( $[\mathrm{RB}]=5 \mu \mathrm{g} / \mathrm{mL}$ ) for another 2 hours. Then, each dish was washed with PBS three times, and paraformaldehyde (4\%) was added to fix the cell morphology. After staining with DAPI, confocal fluorescence images of the cells were acquired by confocal laser scanning microscope (CLSM) in the two channels relevant to DAPI (405 nm) and RB (550 nm).

The cellular uptake of the nanosheets by cells were also measured by fluorescence activated cell sorting (FACS). A549 and HELF cells pre-seeded in six-well plates were incubated with 0 or $5 \mathrm{mg} / \mathrm{mL} \mathrm{HA}$ for 2 hours. The cells were washed with PBS three times and then incubated with NGO-SS-HA-RB ([RB] $=5 \mu \mathrm{g} / \mathrm{mL})$ for another 2 hours. After washing with PBS three times, the cells were harvested with trypsin and re-suspended in PBS for FACS analyses.

\section{Redox-dependent intracellular cargo release}

To clarify the mechanism of intracellular drug release from the nanosheets, RB was again used as the fluorescent probe. 
Briefly, A549 cells were seeded into a confocal dish at a density of $1 \times 10^{5}$ for 24 hours. Next, the cells were treated with fresh cell medium containing GSH-OEt at various concentrations for 2 hours and incubated with NGO-SS-HA-RB $([\mathrm{RB}]=5 \mu \mathrm{g} / \mathrm{mL})$ for another 2 hours. After washing with PBS twice, fluorescence images of the cells were acquired by CLSM.

For the FACS analyses, A549 cells pre-seeded in six-well plates were treated with GSH-OEt for 2 hours, followed by incubation with NGO-SS-HA-RB ([RB] $=5 \mu \mathrm{g} / \mathrm{mL})$ for another 2 hours. These cells were analyzed by flow cytometry after trypsinization and washing with PBS three times.

\section{Evaluation of intracellular Gef levels}

A549 cells were divided into four groups: group 1 with cells only, group 2 with cells incubated with HA+ NGO-SS-HAGef, group 3 with cells treated with NGO-SS-HA-Gef only, and group 4 with cells incubated with GSH-OEt+ NGO-SSHA-Gef. After the corresponding treatments, the cells were washed with PBS three times, harvested after trypsinization, and centrifuged at 1,500 rpm for 10 minutes. Then, the cells were re-suspended in $1 \mathrm{~mL}$ of methanol, sonicated, and centrifuged at 15,000 rpm. Finally, the supernatants were evaluated by an absorbance spectrometer to determine the concentration of Gef.

\section{Therapy in vitro}

In the current work, the CCK-8 assay was used to investigate the cytotoxic effects of the NGO-SS-HA-Gef nanosheets on A549 cells. First, A549 cells pre-seeded into 96-well plates were incubated with 0 or $10 \mathrm{mM} \mathrm{GSH}-\mathrm{OEt}$ for 2 hours and washed with PBS three times. NGO-SS-HA-Gef with various Gef concentrations was added to these 96-well plates and incubated with the cells for 24, 48, and 72 hours. The absorbance of each well was measured at $450 \mathrm{~nm}$ on a microplate reader to determine the cell viability.

\section{Detection of the cell cycle and cell apoptosis}

To further study the therapeutic efficacy of the nanosheets, the cell cycle and cell apoptosis after various treatments were analyzed by FACS. For the cell cycle assay, A549 cells preseeded in 6-well plates were treated with 0 or $10 \mathrm{mM} \mathrm{GSH}$ OEt for 2 hours and washed with PBS three times. The cells were incubated with Gef or NGO-SS-HA-Gef at the same Gef concentrations for 24, 48, and 72 hours. After trypsinization and washing twice with ice-cold PBS, the cells were fixed with cold $70 \%$ ethanol for 24 hours at $4{ }^{\circ} \mathrm{C}$. Subsequently, the fixed cells were washed with PBS twice by centrifugation at
$1,500 \mathrm{rpm}$ and stained with a solution containing $1 \%(\mathrm{v} / \mathrm{v})$ Triton X-100, 0.01\% RNase, and $0.05 \%$ propidium iodide (PI) for 30 minutes in the dark for flow cytometry analyses.

For the detection of cell apoptosis, A549 cells pre-seeded in six-well plates were treated with or without $10 \mathrm{mM} \mathrm{GSH}-$ OEt for 2 hours. After washing with PBS, fresh cell medium containing Gef or NGO-SS-HA-Gef $([\mathrm{Gef}]=10 \mu \mathrm{g} / \mathrm{mL})$ was added and incubated with the cells for 24, 48, and 72 hours. Then, the cells were trypsinized, washed with PBS, and stained with fluorescein isothiocyanate-labeled Annexin V and PI. The percentage of apoptotic cells was quantified using flow cytometry.

\section{Tissue biodistribution in vivo}

All studies involving animals were carried out in accordance with the NSFC regulation concerning the care and use of experimental animals approved by the Institutional Animal Care and Use Committee of Fuzhou University.

The tissue biodistribution of NGO-SS-HA-Gef was evaluated in mice bearing lung tumors and compared with that of free Gef. To develop the tumor model, A549 cells $\left(5 \times 10^{6}\right)$ suspended in $50 \mu \mathrm{L}$ of PBS were subcutaneously injected into the forelimbs of female $\mathrm{BALB} / \mathrm{c}$ nude mice. When the mean tumor volume reached $\sim 200 \mathrm{~mm}^{3}$, the nude mice were randomly divided into six groups ( $n=3 /$ group) and treated with NGO-SS-HA-Gef or free Gef (dissolved in PBS containing $0.5 \%$ DMSO) at a dose of $20 \mu \mathrm{g} / \mathrm{mL}$ Gef. At designated time points (1,2, and 12 hours), all mice were sacrificed, and tissue samples (heart, liver, spleen, lung, kidney, and tumor) were collected and weighed. The concentration of Gef in tissue samples was determined by HPLC. The abovementioned samples (100 mg) were ground with $1 \mathrm{~mL}$ of methanol, and $100 \mu \mathrm{L}$ of the homogenate was mixed with $900 \mu \mathrm{L}$ of internal standard solution $(0.1 \mu \mathrm{g} / \mathrm{mL}$ erlotinib in methanol) for HPLC analysis with a $\mathrm{C} 18$ column $(4.6 \times 150 \mathrm{~mm}, 5 \mathrm{~mm})$. The mobile phase was methanol/ ammonium acetate aqueous solution $=70: 30(\mathrm{v} / \mathrm{v})$, and the detection wavelength was $343 \mathrm{~nm}$.

\section{Therapy in vivo}

To study the therapeutic efficacy of NGO-SS-HA-Gef in depth, HA-modified GO nanosheets with HA that cannot be separated in high-concentration GSH solution were developed as a control. HA was conjugated onto the surface of NGO with HMD via EDC/NHS method. Gef was loaded on to NGO-HMD-HA using a similar method as the loading of Gef onto NGO-SS-HA.

The tumor model was developed as described earlier. After the mean tumor volume reached $\sim 100 \mathrm{~mm}^{3}$, the nude 
mice were randomly divided into five groups ( $\mathrm{n}=5 /$ group). Each group of nude mice was intravenously injected with $200 \mu \mathrm{L}$ of PBS containing 0.5\% DMSO, Gef, NGO-HMDHA-Gef, or NGO-SS-HA-Gef ([Gef] $=20 \mu \mathrm{g} / \mathrm{mL}$ ) every 3 days for 24 days. The tumor sizes were measured by a Vernier caliper and calculated as follows: $\mathrm{V}\left(\right.$ volume, $\left.\mathrm{mm}^{3}\right)=$ length $(\mathrm{mm}) \times$ width $^{2}\left(\mathrm{~mm}^{2}\right) / 2$. After 24 days, all the mice were sacrificed, and the major organs were collected for hematoxylin and eosin (H\&E) and immunohistochemical (IHC) staining.

\section{Results and discussion Synthesis of NGO-SS-HA}

GO was synthesized from graphite flakes using the improved Hummer's method, ${ }^{32}$ and NGO was obtained by filtering through a $0.45 \mu \mathrm{m}$ filter. NGO was stable in pure water for a month but aggregated in the presence of salts, especially in PBS (Figure 1B). In this study, HA, a type of hydrophilic mucopolysaccharide, was conjugated with NGO to enhance the stability of NGO in PBS and cell medium. However, we found that HA could generate a shell barrier on the surface of NGO after conjugation, which limited drug release. To address this problem, we conjugated HA with $\mathrm{NGO}$ via a disulfide linker. The resulting NGO-SS-HA not only was evenly dispersed in PBS and cell medium but also accelerated the drug release in response to tumor-relevant GSH.

Characterization of GO was achieved by X-ray diffraction (Figure S1) and Raman spectroscopy (Figure S2). After carboxylation of $\mathrm{GO}$, the oxygen content was found to increase from $51.36 \%$ to $58.87 \%$ (Figure $\mathrm{S} 3$ ), and the zeta potential of GO also changed from -37.5 to $-53.0 \mathrm{mV}$ (Figure $\mathrm{S} 4$ ), owing to the conversion of the $\mathrm{OH}$ groups into $\mathrm{COOH}$ groups. When HA was conjugated with NGO by a disulfide bond, the Fourier transform infrared (FTIR) spectrum confirmed the presence of HA on the NGO nanosheets (Figure 1C). Briefly, the FTIR spectrum of HA-SS showed peaks at $1,658 \mathrm{~cm}^{-1}$ and 1,560 $\mathrm{cm}^{-1}$ associated with amide bonds, confirming that cystamine dihydrochloride was coupled with HA through amide bonds. Next, HA-SS was conjugated with NGO via the EDC/NHS method. The spectrum showed disappearance of the peak at $1,736 \mathrm{~cm}^{-1}$ for NGO and the presence of the characteristic peaks of HA-SS at $1,318 \mathrm{~cm}^{-1}$ and $1,150 \mathrm{~cm}^{-1}$ (skeletal acetal valence band), suggesting successful coupling of HA on the surface of NGO. UV-vis spectroscopy is also a good technique for the characterization of NGO-SS-HA (Figure S5).

We then used AFM to characterize NGO nanosheets before and after HA functionalization (Figure 1D and E).
As-prepared NGO nanosheets were found to have an average thickness of $\sim 1 \mathrm{~nm}$, suggesting the complete exfoliation of the NGO nanosheets down to individual ones. ${ }^{33}$ After HA functionalization, the average thickness of the NGO nanosheets increased to $\sim 2.4 \mathrm{~nm}$ (Figure 1G), whereas their average diameter decreased from $\sim 250$ to $\sim 125 \mathrm{~nm}$ (Figure $1 \mathrm{~F}$ and $\mathrm{H})$, mainly because of sonication.

\section{Drug loading and redox-responsive drug release in vitro}

$\mathrm{GO}$, a 2D material, has the advantage of an ultrahigh surface area, ${ }^{34-36}$ which facilitates the loading of Gef onto nanosheets. To investigate the potential Gef loading ability of NGOSS-HA nanosheets, NGO-SS-HA solution $(0.4 \mathrm{mg} / \mathrm{mL})$ was mixed with various concentrations of Gef and stirred for 24 hours at $\mathrm{pH}$ 7.0. UV-vis spectroscopy was employed to determine the Gef loading efficiency. The absorbance of NGO-SS-HA-Gef in $343 \mathrm{~nm}$ (Figure 2A) increased with increasing weight ratio (between Gef and NGO-SS-HA). When the ratio rose from 2 to 2.5 , the absorbance was found to not increase further (Figure $2 \mathrm{~B}$ ), indicating that maximum drug loading was reached and was $\sim 13.8 \%$.

Many experimental factors are involved in drug release from nanocarriers, ${ }^{37}$ such as nanoparticle size, $\mathrm{pH}$, temperature, redox response, and drug-carrier interactions. To study the behavior of Gef release from the nanosheets, we investigated the drug release change as the function of $\mathrm{pH}$ and GSH concentration through a dialysis method. At a $\mathrm{pH}$ of 5.5 without GSH, $30.8 \%$ of Gef was released from the nanosheets over 72 hours (Figure 2C). Upon exposure to $10 \mathrm{mM} \mathrm{GSH}, 60.1 \%$ of Gef was released from the nanosheets under the same conditions, and the rate of drug release was 1.95 times faster than that in the absence of GSH, suggesting that the GSH level in tumor cells can trigger Gef release from NGO-SS-HA-Gef. When 1 or $0.1 \mathrm{mM} \mathrm{GSH}$ was present in the release buffer, NGO-SS-HA-Gef also exhibited redox-responsive release with a cumulative percent release of $>44.1 \%$ or $39.2 \%$ over 72 hours, respectively. Interestingly, we also found that the cumulative drug release with $10 \mu \mathrm{M}$ GSH was similar to that without GSH, indicating that the linkages between HA and NGO in NGO-SS-HA-Gef would be relatively stable in vivo in the circulation.

In the control experiments performed at $\mathrm{pH} 7.4$ (Figure 2D), drug release acceleration was also observed in the presence of various concentrations of $\mathrm{GSH}$, but the cumulative release curve of Gef over 72 hours was similar to that at $\mathrm{pH} 7.4$ under the same conditions, suggesting that $\mathrm{pH}$ had almost no effect on Gef release from the nanosheets. 
A
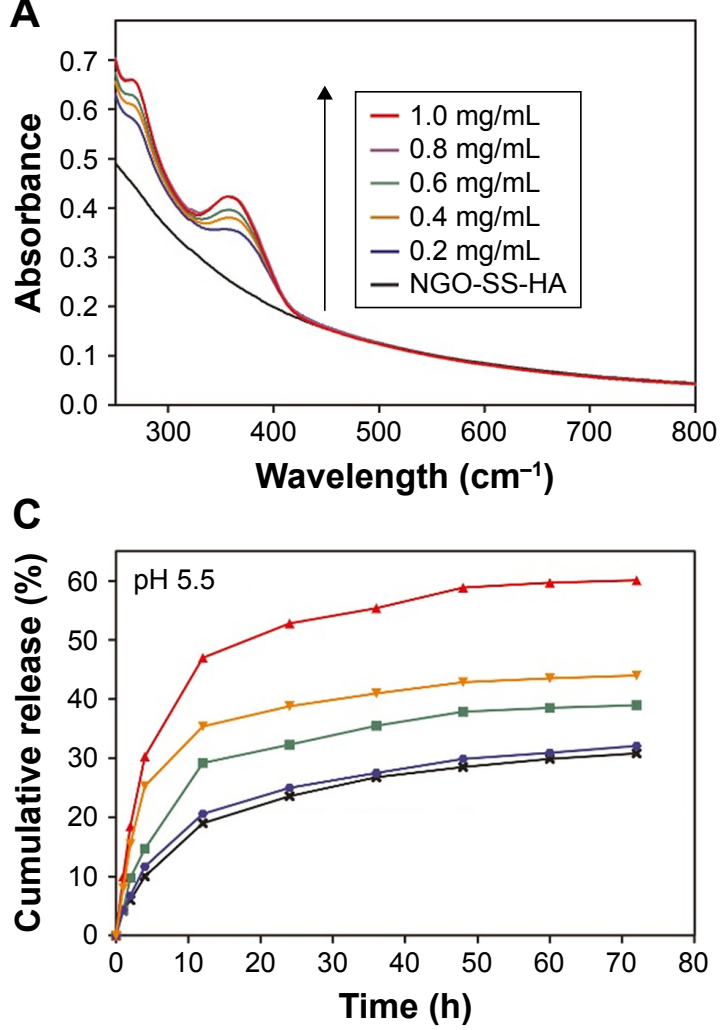

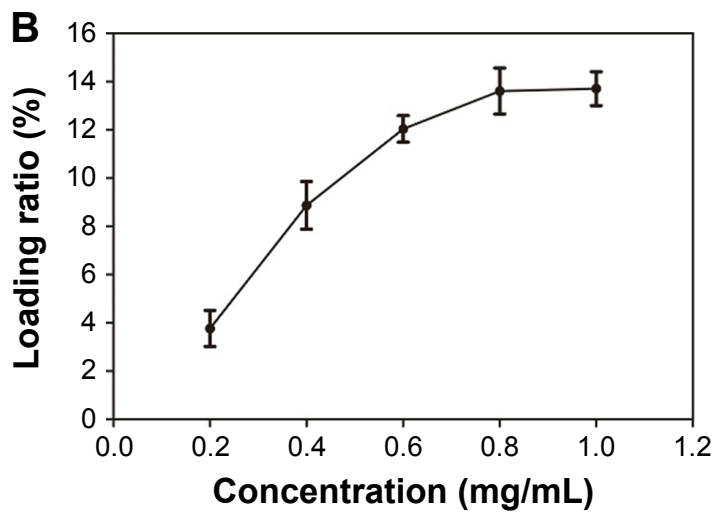

D

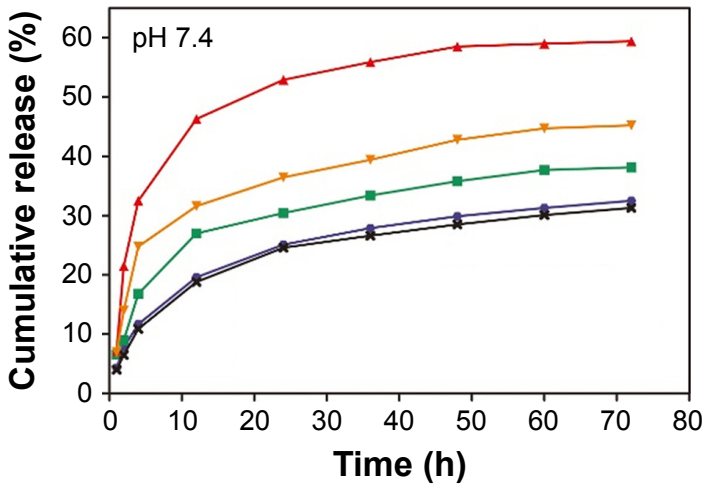

$-10 \mathrm{mM} \mathrm{GSH} \rightarrow 1 \mathrm{mM} \mathrm{GSH} \rightarrow 0.1 \mathrm{mM} \mathrm{GSH} \rightarrow 0.01 \mathrm{mM} \mathrm{GSH} * 0 \mathrm{mM} \mathrm{GSH}$

Figure 2 (A) UV-vis spectra of NGO-SS-HA-Gef obtained at different drug loading concentrations after the removal of free Gef. (B) Plots of the ratio of Gef loaded onto NGO-SS-HA at various concentrations of Gef. (C, D) Gef release from NGO-SS-HA-Gef at pH 5.5 and 7.4 in the presence of GSH as a function of time. Abbreviations: NGO, nano-graphene oxide; HA, hyaluronic acid; Gef, gefitinib; GSH, glutathione.

Therefore, the abovementioned results illustrated that NGOSS-HA-Gef can be used to limit Gef release during blood circulation and accelerate intracellular drug release in tumor cells based on its redox responsiveness to GSH.

\section{Biocompatibility in vitro}

Biocompatibility is an essential concern in the development of nanomaterials for biomedical applications. ${ }^{38}$ Therefore, evaluating the cytotoxicity of NGO-SS-HA was necessary. After incubation with high concentrations of NGO-SS-HA for 72 hours, $>88.0 \%$ of the A549 cells remained alive, even when the nanosheet concentration reached $400 \mu \mathrm{g} / \mathrm{mL}$ (Figure 3A). Similar results were observed for HELF cells (Figure 3B), further confirming that NGO-SS-HA had low cytotoxicity against cells.

In addition, a hemolysis assay was employed to evaluate the blood compatibility of NGO-SS-HA. After various treatments, we found that the hemolysis percentage of NGO-SS-HA was below $4 \%$ at nanosheet concentrations ranging from 25 to $400 \mu \mathrm{g} / \mathrm{mL}$ (Figure 3C), indicating that NGO-SS-HA had admirable blood compatibility with cells.
As a result, the low cytotoxicity and hemolytic activity of the NGO-SS-HA nanosheets contribute to its application in biological fields.

\section{Cellular uptake and intracellular drug release}

The cellular association of the nanosheets was determined using $\mathrm{RB}$ as a fluorescent probe. ${ }^{39} \mathrm{RB}$ was loaded onto the surface of NGO-SS-HA through $\pi-\pi$ stacking and hydrophobic interactions, and a new peak at $575 \mathrm{~nm}$ appeared in the UV-vis spectra (Figure S6). Figure S7 shows that the fluorescence of aqueous NGO-SS-HA-RB solution was weaker than that of free $\mathrm{RB}$ at the same $\mathrm{RB}$ concentration, suggesting partial fluorescence quenching caused by fluorescence resonance energy transfer. ${ }^{40}$

To verify the targeting specificity, both A549 (CD44 receptor-positive) and HELF cells (CD44 receptor-negative) were incubated with NGO-SS-HA-RB for 2 hours, and confocal images of these cells were obtained by CLSM at an excitation wavelength of $550 \mathrm{~nm}$. A strong red fluorescence was evident in A549 cells after incubation with 
A

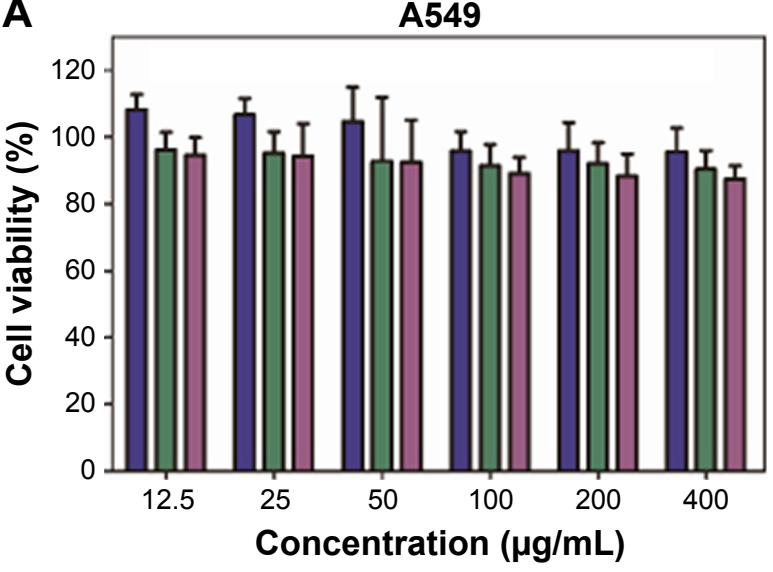

B
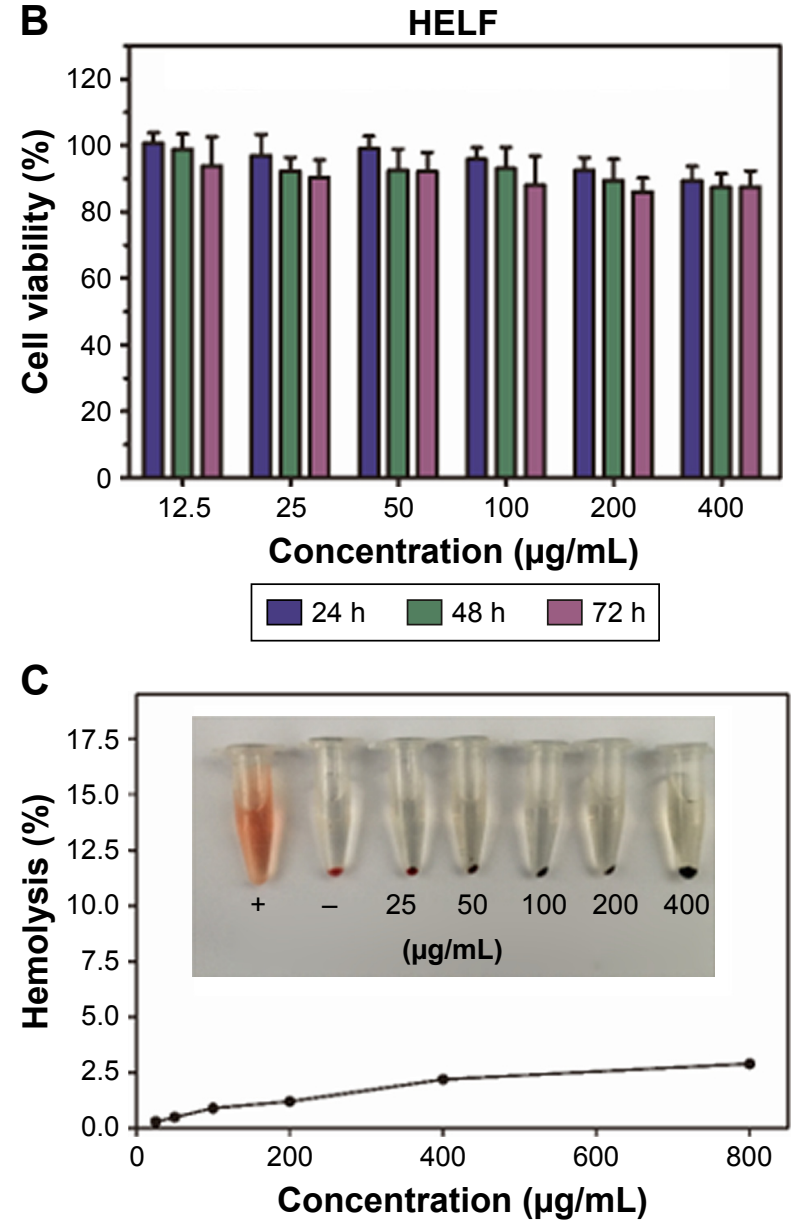

Figure 3 Cell viability of (A) A549 cells and (B) HELF cells incubated with various concentrations of NGO-SS-HA from 24 to 72 hours. (C) Hemolysis percentage of RBCs incubated with NGO-SS-HA at various concentrations for 2 hours. Inset: image of direct observation of hemolysis by NGO-SS-HA.

Abbreviations: NGO, nano-graphene oxide; HA, hyaluronic acid; Gef, gefitinib; h, hours.

NGO-SS-HA-RB, but a very weak fluorescence signal was detected in HELF cells (Figure 4A). Upon pre-treatment with HA, the fluorescence of A549 cells was greatly reduced, suggesting that the cellular uptake of NGO-SS-HA-RB was associated with HA CD44-receptor-mediated endocytosis and could be blocked by excess free HA. The fluorescence intensity of these cells was also quantitatively detected by FACS. As expected, the results of flow cytometry (Figure 4B and C), consistent with the CLSM data, further confirmed that the uptake of NGO-SS-HA by A549 cells was facilitated by the specific interaction between CD44 receptor and HA.

To study the redox-responsive property of the nanosheets with disulfide linkages, GSH-OEt was included in the drug release experiment. ${ }^{26}$ GSH-OEt, a derivative of GSH, had no cytotoxicity to A549 cells (Figure S8) and could act as an external enhancer of the cellular GSH level. A549 cells were pre-treated with various concentrations of GSH-OEt $(0,0.01,0.1,1$, and $10 \mathrm{mM})$ for 2 hours, incubated with NGOSS-HA-RB $([R B]=5 \mu \mathrm{g} / \mathrm{mL})$ for another 2 hours, and then imaged by CLSM (Figure 4D). We found that the intracellular red fluorescence became increasingly strong with increasing GSH-OEt concentration, indicating that more RB was released from the nanosheets in the presence of GSH, mainly because of the removal of the HA shell via the cleavage of disulfide bonds caused by GSH in a dose-dependent manner. Furthermore, we also studied GSH-dependent cellular RB release by FACS. Similar to the CLSM results, FACS (Figure 4E) revealed that stronger fluorescence intensity could be observed in the cells pre-treated with $10 \mathrm{mM} \mathrm{GSH}-$ OEt than the cells pre-treated with lower concentrations of GSH-OEt. These results suggested that the drug release from NGO-SS-HA could be accelerated by GSH-mediated degradation of the disulfide linkages between HA and NGO.

Based on the abovementioned results, we directly investigated the concentration of intracellular Gef by UVvis spectroscopy. As shown in Figure 4F, cells incubated with NGO-SS-HA-Gef contained substantially more Gef than cells pre-incubated with HA. More importantly, upon pre-incubation of cells with $10 \mathrm{mM} \mathrm{GSH-OEt}$, the intracellular concentration of Gef increased and was 2.05 times higher than that in the absence of GSH-OEt under the same conditions, consistent with the drug release results in vitro. Therefore, the results indicated that NGO-SS-HA-Gef could not only specially bind with CD44 receptor-overexpressing cancer cells but also accelerate Gef release from intracellular nanosheets based on its redox responsiveness to GSH.

\section{Anti-proliferative assay in vitro}

To study the cytotoxicity of NGO-SS-HA-Gef to cancer cells in vitro, we incubated A549 cells with NGO-SS-HA-Gef at various Gef concentrations for 24,48 , and 72 hours and then investigated cell viability by the CCK- 8 assay. As shown in 
Figure 5A, both free Gef and NGO-SS-HA-Gef inhibited the proliferation of A549 cells in a dose-dependent manner. NGO-SS-HA-Gef exhibited a greater cytotoxic effect on A549 cells than free Gef at the same Gef concentration, possibly due to HA-targeting-enhanced cellular uptake of Gef provided by the nanosheets. After the addition of GSHOEt, NGO-SS-HA-Gef showed the strongest cytotoxicity, revealing that redox responsiveness may be an effective strategy to accelerate drug release in GSH-rich environments, thus resulting in enhanced antitumor activity. In addition, the effect of incubation time on the cytotoxicity of NGOSS-HA-Gef was also investigated. The cytotoxicity of both Gef and NGO-SS-HA-Gef against A549 cells was found to become stronger with increasing incubation time (Figure $5 \mathrm{~B}$ and C). Moreover, NGO-SS-HA-Gef in the presence of GSH exhibited the strongest anti-proliferative effect among the test groups. Therefore, we determined that NGO-SS-HA could function as an excellent nanocarrier of Gef by distinctly enhancing its cytotoxicity through CD44 receptor-mediated endocytosis and by accelerating drug release based on the redox responsiveness of the carrier to GSH.

\section{Cell cycle and cell apoptosis}

The effects of NGO-SS-HA-Gef on cell proliferation may be explained in part by its effects on the cell cycle. ${ }^{41}$ Therefore, we treated A549 cells with NGO-SS-HA-Gef in a timedependent manner and then analyzed the cell cycle. The percentage of A549 cells treated with Gef for 24 hours in the $\mathrm{G} 0 / \mathrm{G} 1$ phase was $\sim 60.53 \%$, which was lower than that of the controls, indicating that Gef can cause G0/G1 arrest of A549 cells (Figure 6A). Following treatment of the A549 cells with NGO-SS-HA-Gef at the same Gef concentration, the percentage of cells in the G0/G1 phase increased to $\sim 71.21 \%$, indicating that NGO-SS-HA-Gef can enhance Gef-induced G0/G1 arrest. In other words, NGO-SS-HAGef could better prevent the cells from entering the $\mathrm{S}$ phase, during which some proteins involved in DNA replication are copied and thus inhibit cell proliferation. When the cells were
A

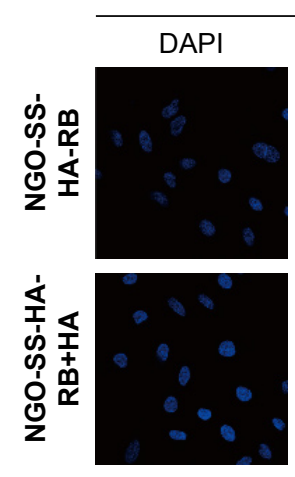

B

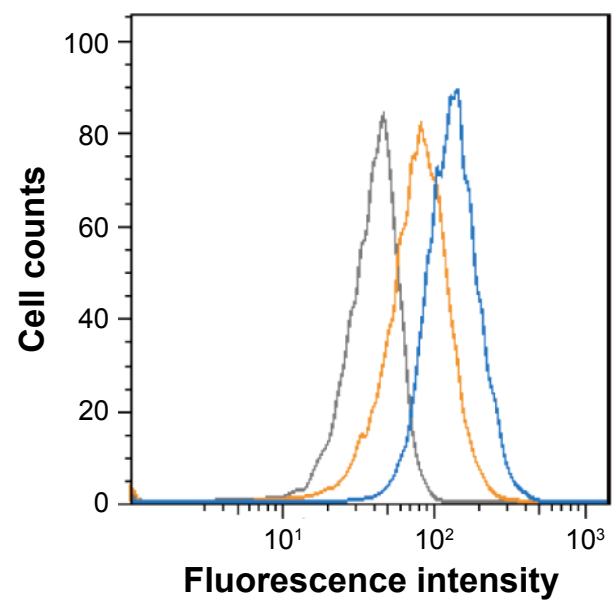

A549

A549
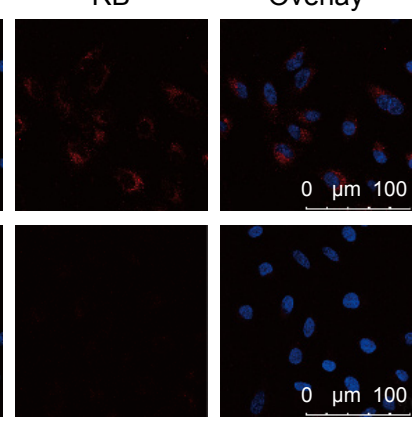

- Control - NGO-SS-HA-RB + HA - NGO-SS-HA-RB
HELF

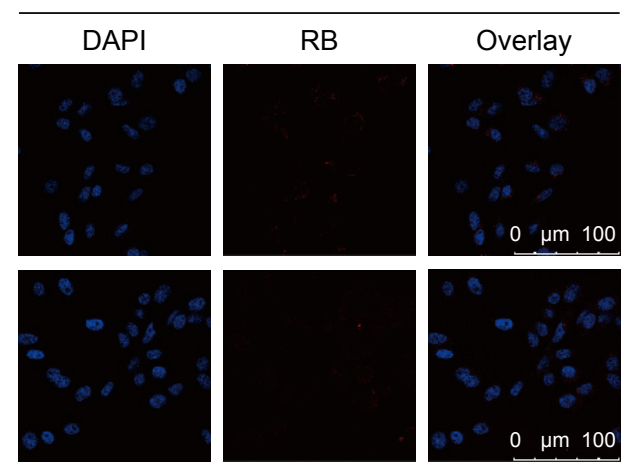

C

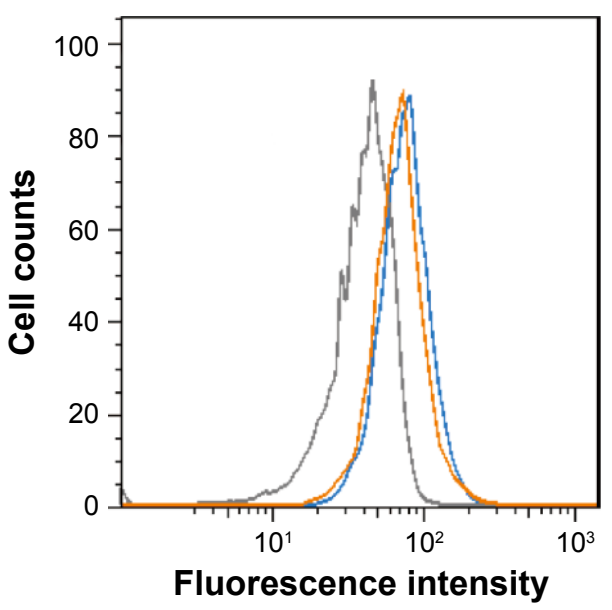

Figure 4 (Continued) 
D
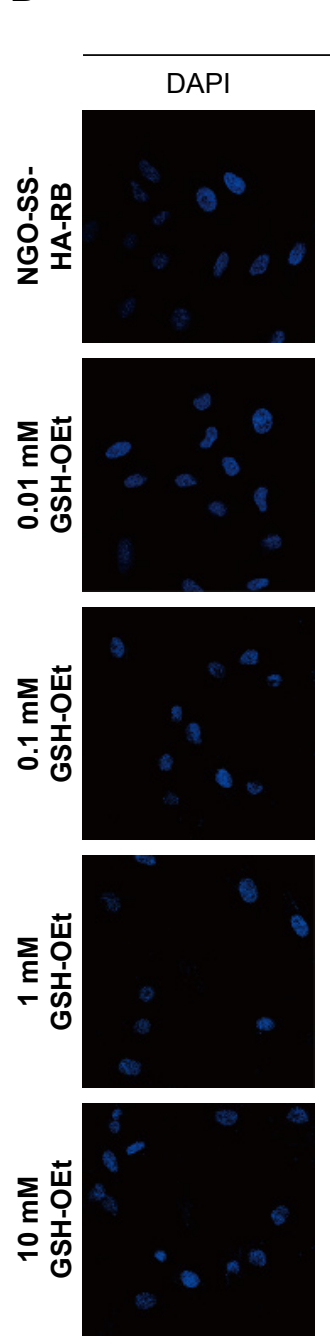

A549
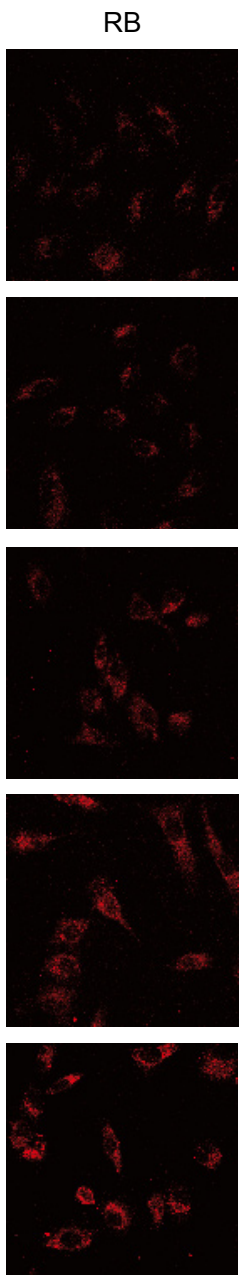

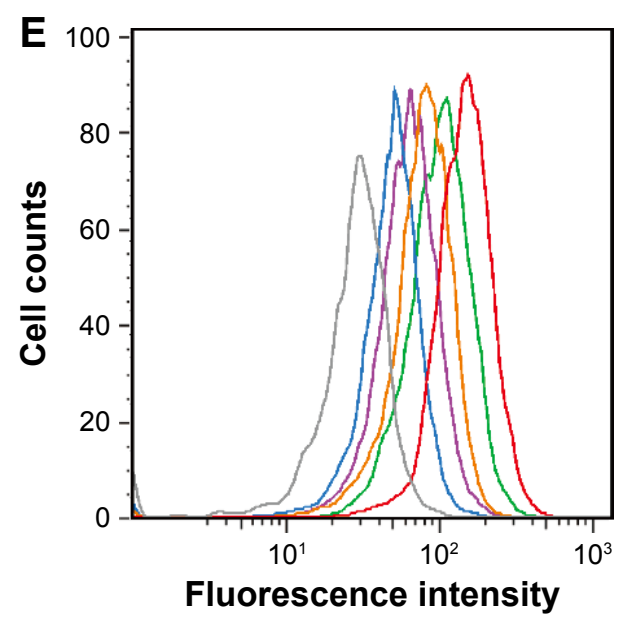

$0 \mu \mathrm{m} 100$
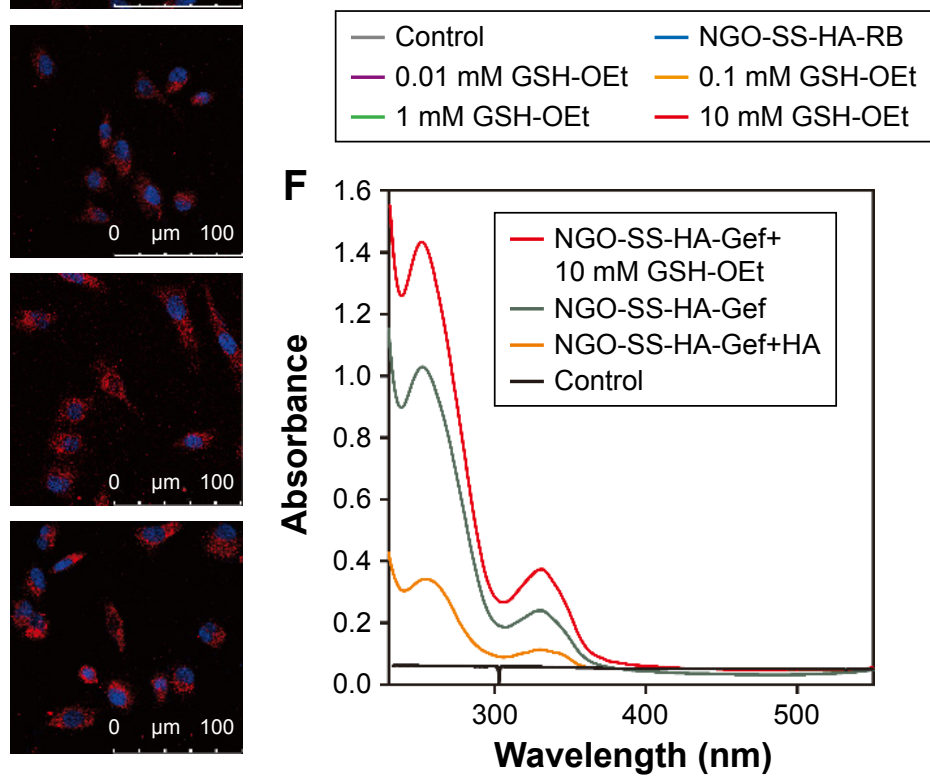

Figure 4 Confocal images (A) and flow cytometric analysis (B and C) of A549 and HELF cells with or without pre-treatment with excess HA incubated with NGO-SS-HARB for 2 hours. Confocal images (D) and flow cytometric measurement (E) of RB release from in A549 cells pre-treated with various concentrations of GSH-OEt; UV-vis spectra (F) of intracellular Gef concentration after A549 cells were treated with various treatments.

Abbreviations: NGO, nano-graphene oxide; HA, hyaluronic acid; Gef, gefitinib; RB, rhodamine B; GSH-OEt, glutathione reduced ethyl ester.

pre-incubated with $10 \mathrm{mM} \mathrm{GSH}-\mathrm{OEt}$, the percentage of cells in the $\mathrm{G} 0 / \mathrm{G} 1$ phase continued to increase from $\sim 71.21 \%$ to $\sim 79.83 \%$, suggesting that NGO-SS-HA-Gef more strongly arrested cells in the G0/G1 phase due to accelerated drug release based on its redox responsiveness to GSH. On the other hand, after increasing the incubation time, we found (Figure 6B and C) that the percentage of cells in the G0/G1 phase in each group increased in a time-dependent manner. Together with the abovementioned results, NGO-SS-HAGef was found to enhance G0/G1 arrest of A549 cells in a time-dependent manner, especially in the presence of GSH, thus preventing cells from progressing to the $\mathrm{S}$ phase and inhibiting their proliferation.
To explore whether the cell growth inhibition in vitro observed was due to enhanced apoptosis, the proportion of apoptotic cells was determined by FACS using Annexin V/PI staining. ${ }^{42}$ After incubation with NGO-SS-HA-Gef for 24 hours, the NGO-SS-HA-Gef-treated A549 cells exhibited an apoptotic rate of $\sim 15.02 \%$, which was significantly higher than that in cells treated with free Gef (Figure 6D). After pre-treatment with $10 \mathrm{mM}$ GSH-OEt, the apoptotic rate of cells increased to $\sim 25.42 \%$. The significant cell-killing effect was attributed to the HA-targeting-enhanced cellular uptake of Gef-loaded nanosheets and GSH-triggered drug release. When the incubation time was increased to 48 or 72 hours, the apoptotic rate of each group also increased (Figure 6E 


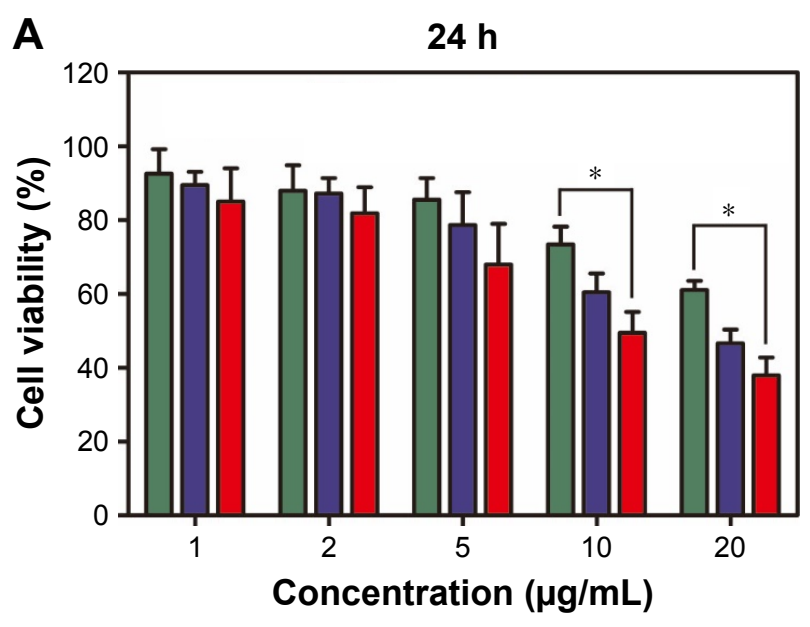

B

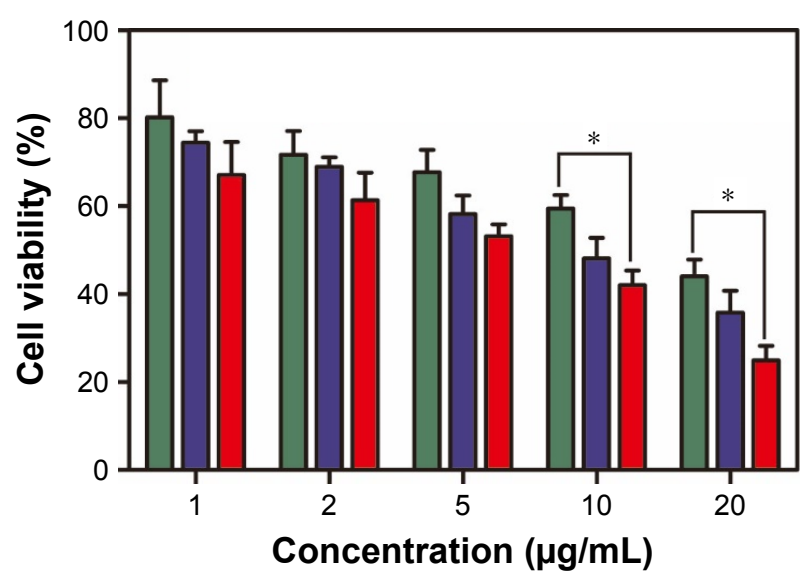

C

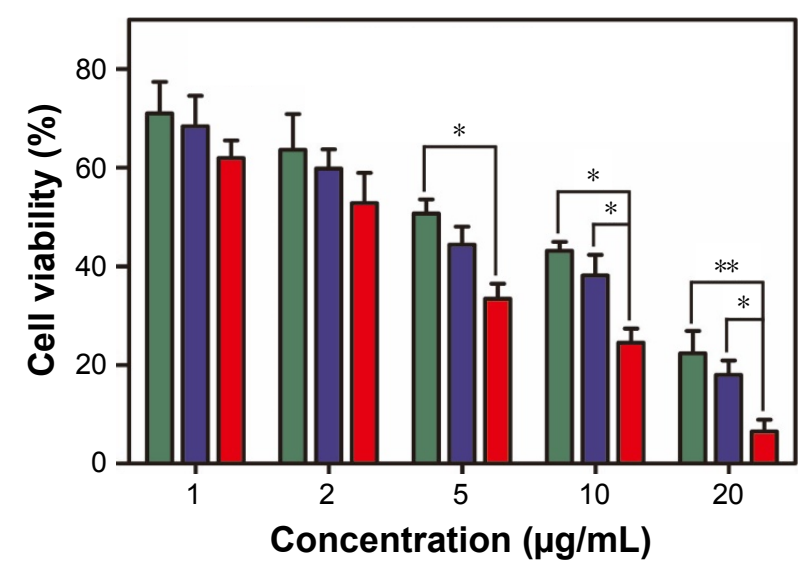

Gefitinib $\square$ NGO-SS-HA-Gef

NGO-SS-HA-Gef + GSH-OEt

Figure 5 Cell viability of A549 cells after incubation with free Gef, NGO-SS-HAGef, or NGO-SS-HA-Gef + 10 mM GSH-OEt for 24 (A), 48 (B), and 72 hours (C). $* P<0.05, * * p<0.0$ I (NGO-SS-HA-Gef + GSH-OEt vs free Gef and NGO-SS-HA-Gef only). The differences between groups were considered statistically significant when the $P$-value was $<0.05$.

Abbreviations: NGO, nano-graphene oxide; HA, hyaluronic acid; Gef, gefitinib; $\mathrm{RB}$, rhodamine $\mathrm{B}$; GSH-OEt, glutathione reduced ethyl ester. and F), suggesting that the effects on the apoptotic rate of A549 cells are time-dependent, which is consistent with the in vitro results of the anti-proliferative assay.

\section{Tissue biodistribution in vivo}

Tissue biodistribution of Gef was assessed in A549 tumorbearing mice. Gef was found to disperse rapidly, that is, within 1 hour, into the major organs of mice (heart, liver, spleen, lung, and kidney) after intravenous injection of free Gef or NGO-SSHA-Gef (Figure 7A). The amount of Gef in the major organs followed the order: liver $>$ lung $>$ spleen $>$ kidney $>$ heart. The Gef contents in major organs reached maximum levels after 2 hours (Figure 7B) and decreased at 12 hours (Figure 7C), mainly owing to the gradual degradation and metabolism of the abovementioned two formulations in vivo.

At all the time points, the Gef content in the tumors of mice after intravenous injection with NGO-SS-HA-Gef was higher than that in the tumors of mice injected with free Gef. In particular, at 2 hours, the Gef level in the tumors of the NGOSS-HA-Gef-treated mice was 2.12 -fold $(P<0.05)$ greater than that in the tumors of the mice treated with free Gef. This result was attributed to the efficient accumulation of NGOSS-HA-Gef in the tumors through a combination of passive (enhanced permeability and retention effect) and active (HA as a targeting moiety for the $\mathrm{CD} 44$ receptor) targeting.

\section{Antitumor efficacy in vivo}

Encouraged by the fascinating therapeutic effect of NGOSS-HA-Gef in vitro, we next conducted comparative efficacy studies in vivo using the nanosheets. After the tumor volume reached $\sim 100 \mathrm{~mm}^{3}$, these nude mice were randomly divided into five groups with five mice per group. The mice were intravenously injected with PBS, NGO-SS-HA, free Gef, NGO-HMD-HA-Gef, or NGO-SS-HA-Gef every 3 days. During 24 days of treatment, the body weight and the tumor volume of each group were recorded every time. The average body weight of each group showed no significant variation (Figure 8A), suggesting that HA-grafted NGO exerted no significant side effects. The mice treated only with NGO-SS-HA experienced rapid tumor volume increases as a function of time, similar to the observations in PBS-treated mice, indicating that NGO-SS-HA induced a negligible effect on tumor growth (Figure $8 \mathrm{~B}$ ). Moreover, compared with free Gef, NGO-HMD-HA-Gef was observed to significantly improve anticancer efficacy, as verified by a lower tumor growth rate. Furthermore, owing to HA CD44receptor-mediated tumor targeting and GSH-triggered drug 

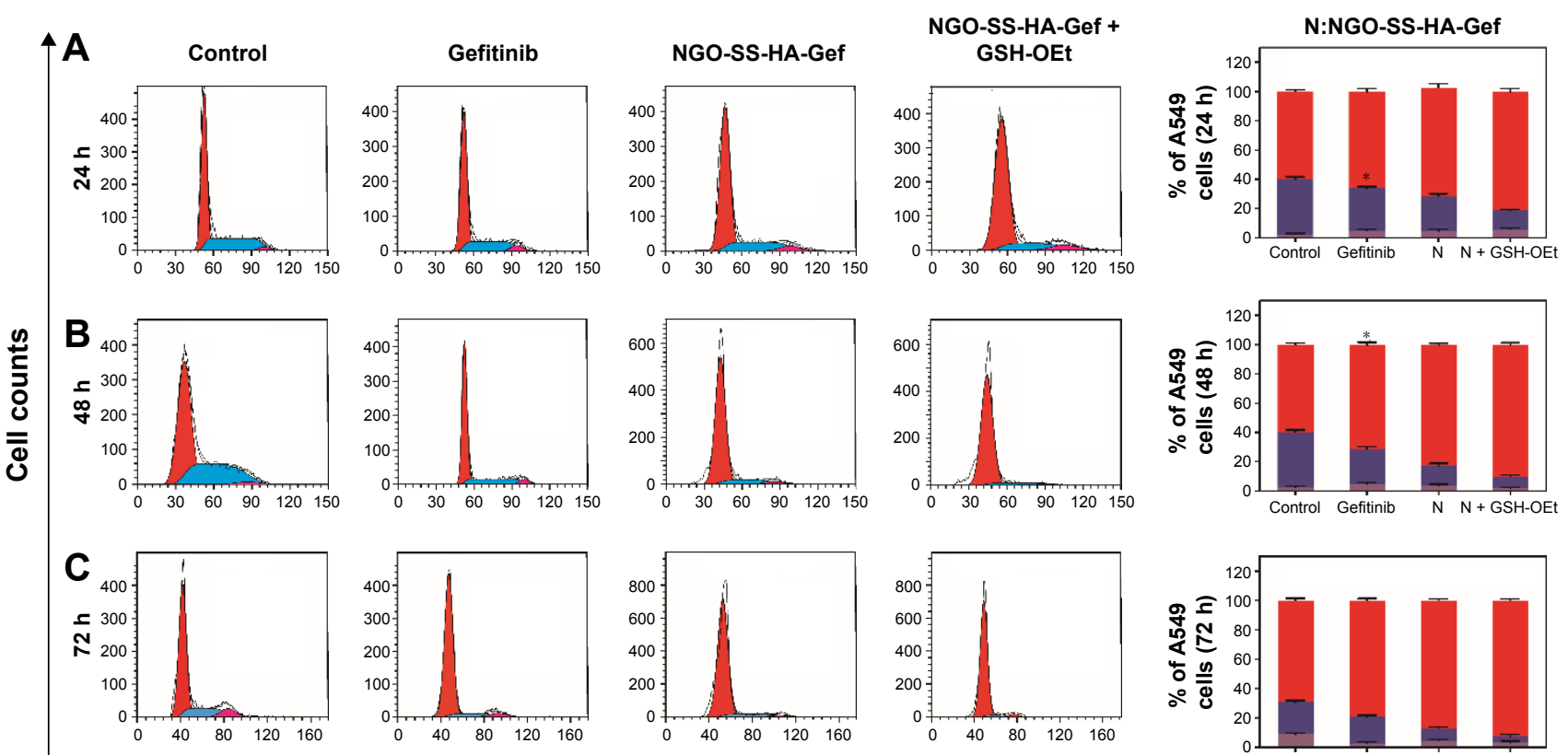

PI-A
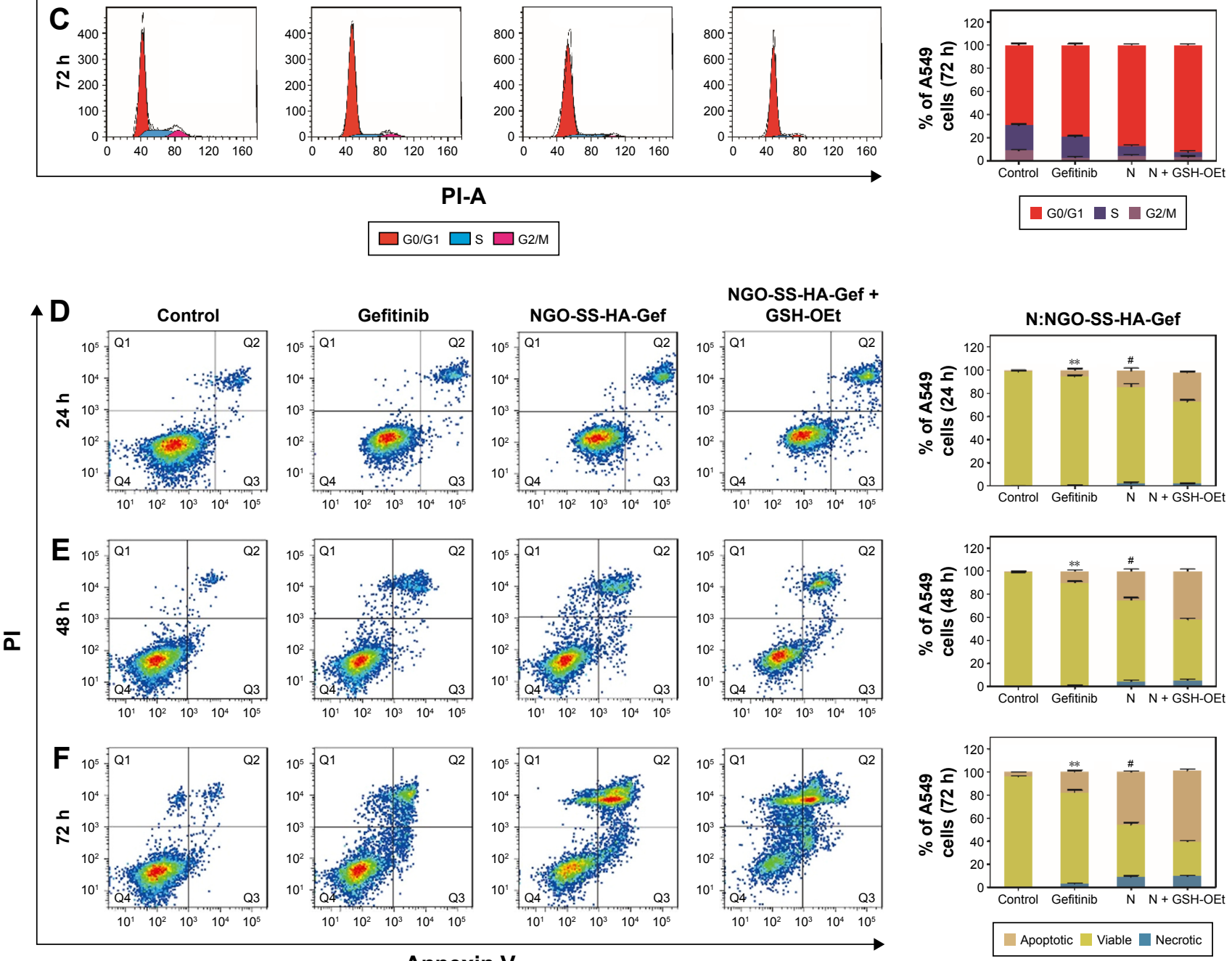

Annexin V

Figure 6 (A-C) Cell cycle distribution (G0/GI, S, and G2/M) was determined after various treatments by flow cytometry. A549 cells were treated with free Gef or NGOSS-HA-Gef $([\mathrm{Gef}]=10 \mu \mathrm{g} / \mathrm{mL})$ in the presence or absence of GSH-OEt. Controls remained untreated. (D-F) Apoptotic cells were detected by staining with FITC-Annexin $\mathrm{V} / \mathrm{Pl}$ after various treatments. Controls remained untreated. $* P<0.05$, $* * P<0.01$ compared with the Gef group. ${ }^{*} P<0.05$ compared with the NGO-SS-HA-Gef only group. Abbreviations: NGO, nano-graphene oxide; HA, hyaluronic acid; Gef, gefitinib; RB, rhodamine B; GSH-OEt, glutathione reduced ethyl ester; FITC, fluorescein isothiocyanate; $\mathrm{Pl}$, propidium iodide.

release, mice treated with NGO-SS-HA-Gef experienced the lowest tumor volume growth among these groups, indicating that NGO-SS-HA-Gef can significantly inhibit the tumor growth, which is consistent with the results of the CCK-8 assay. After 24 days of various treatments, all the mice were sacrificed, and their tumors (Figure 8C) and major organs were collected and sectioned for histological analysis. H\&E staining of the major organs (Figure 8D) showed that there was no appreciable organ damage. The therapeutic efficacy of NGO-SS-HA-Gef in A549 tumor-bearing mice could 

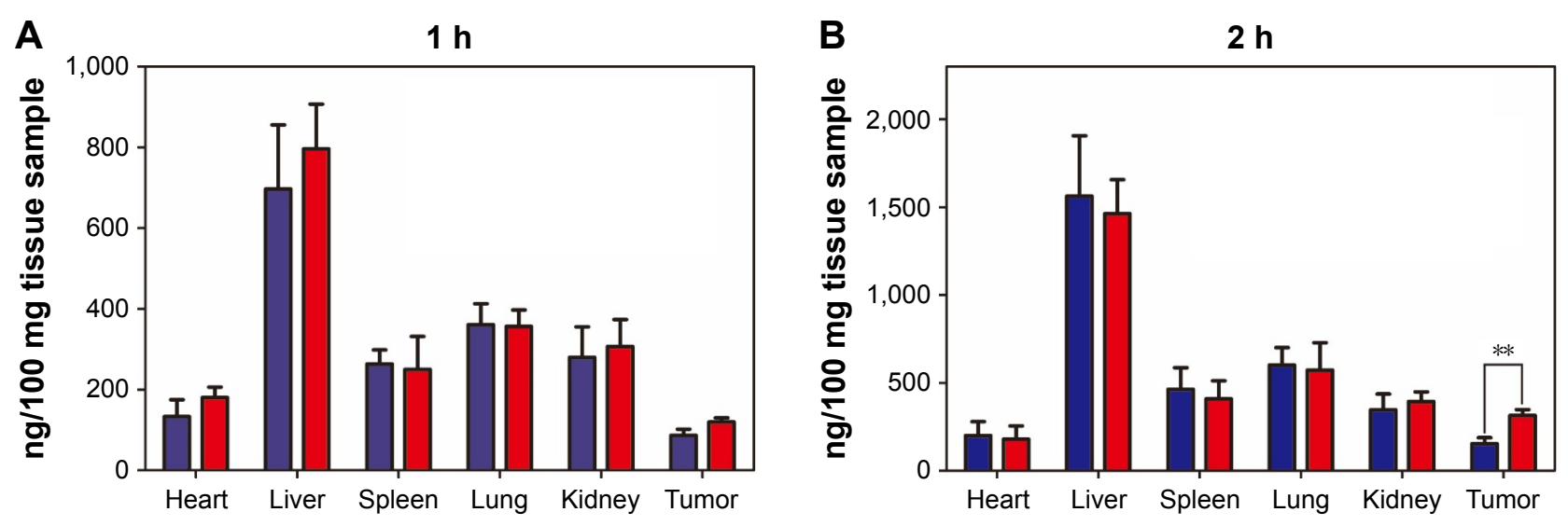

C

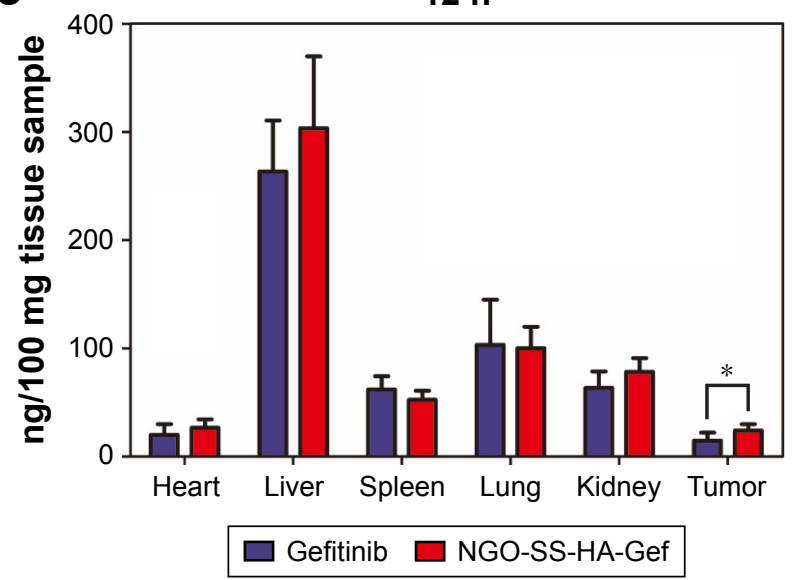

Figure 7 Tissue biodistribution of Gef after intravenous administration of NGO-SS-HA-Gef to A549 tumor-bearing mice: (A) I, (B) 2 and (C) 12 hours following administration, with free Gef used as a control. Error bars were based on three parallel mice. Significant differences between groups are labeled with $* P<0.05$ and $* * P<0.01$.

Abbreviations: NGO, nano-graphene oxide; HA, hyaluronic acid; Gef, gefitinib.

also be detected by H\&E staining of tumors. H\&E staining (Figure 8E) revealed that the PBS group showed vigorous tumor growth, whereas heteromorphosis along with cell necrosis and lysis occurred in tumor cells in the Gef, NGO-
HMD-HA-Gef, and NGO-SS-HA-Gef groups. A large number of dead cells in the tumor were observed in the mice treated with NGO-SS-HA-Gef, indicating excellent antitumor efficacy. Gef, an important EGFR-TK inhibitor, can
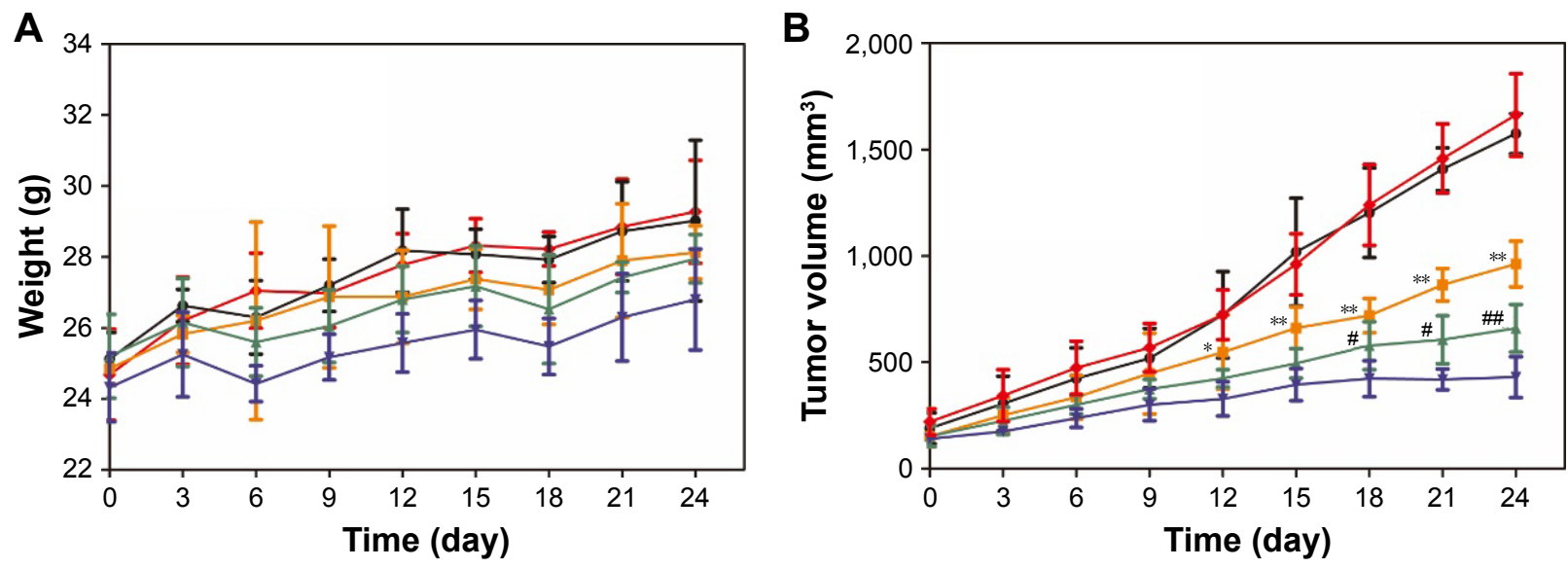

$\multimap$ PBS $\bullet$ NGO-SS-HA $\rightarrow-$ Gefitinib $₫$ NGO-HMD-HA-Gef $\rightarrow$ NGO-SS-HA-Gef

Figure 8 (Continued) 


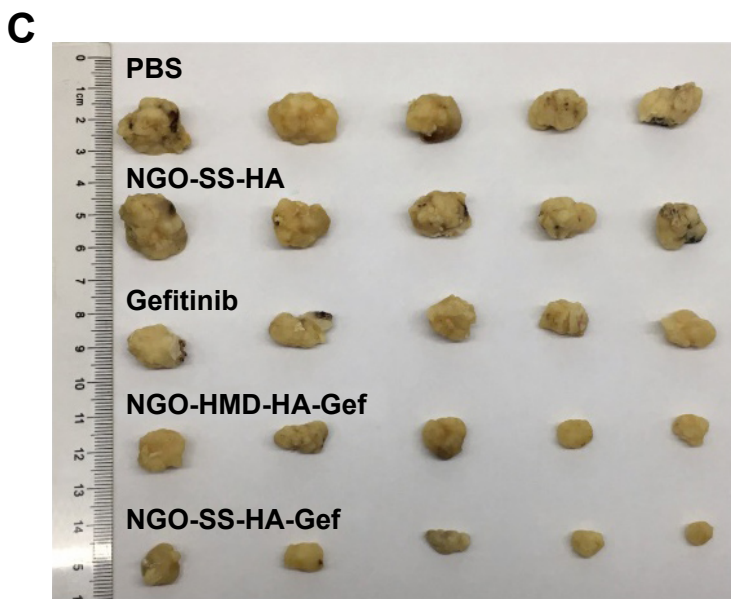

E

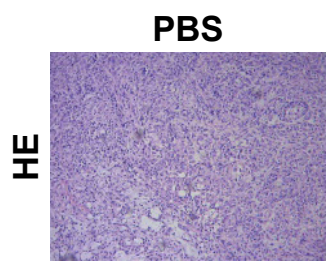

D

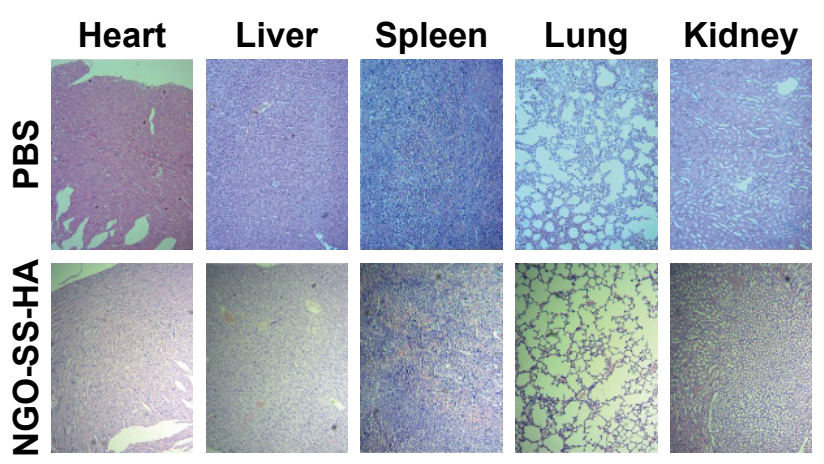

F

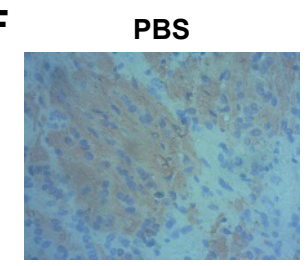

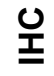

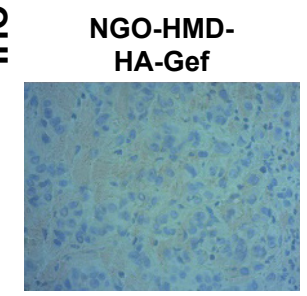

Gefitinib

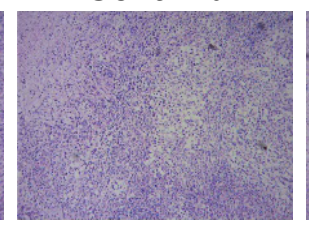

NGO-HMD-

HA-Gef

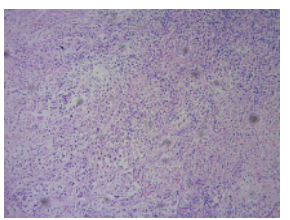

NGO-SS-HA-Gef

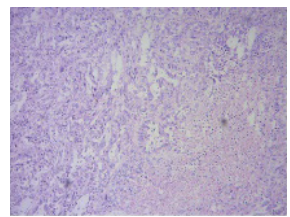

\section{G}
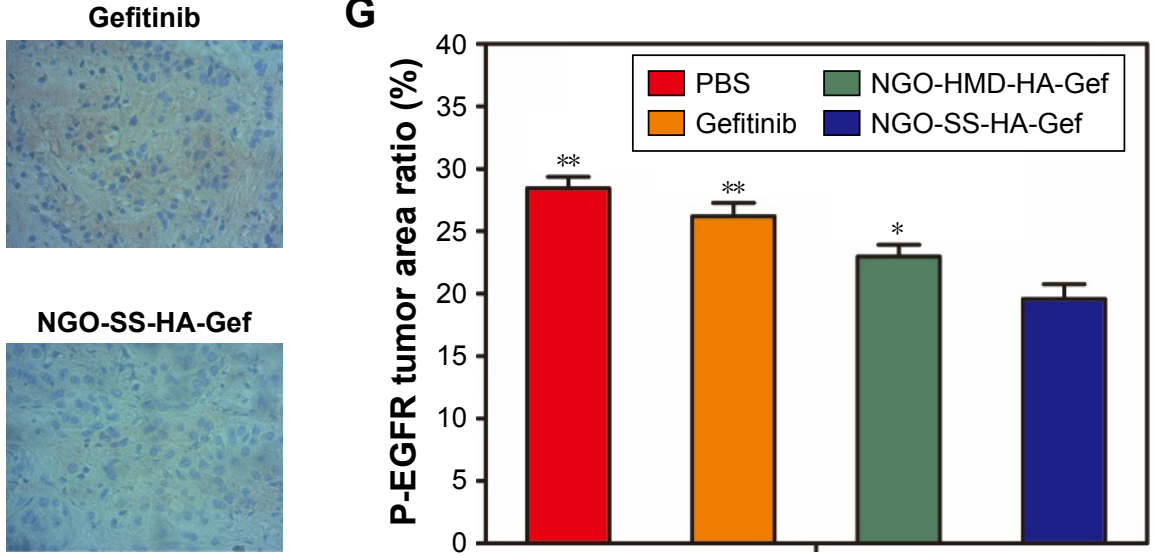

Figure 8 Comparative investigation of tumor inhibitory effects in vivo.

Notes: (A) Relative mouse body weight of each group as a function of time. (B) Tumor growth curves of different groups of mice after various treatments. Significant differences appeared between the NGO-SS-HA-Gef and the free Gef groups and are marked as $* P<0.05$ and $* * P<0.0$ I. Significant differences appeared between the NGO-SS-HA-Gef and NGO-HMD-HA-Gef groups and are labeled with $\# P<0.05$ and $\# P<0.01$. (C) Photographs of tumors from the five groups. (D) H\&E images of major organs from control mice and mice 24 days post-NGO-SS-HA injection. (D and $\mathbf{E}) \mathrm{H} \& \mathrm{E}$ staining of tumors from different groups. (F) IHC analysis of the expression of P-EGFR in mice; P-EGFR was stained brown. (G) Quantification of the P-EGFR expression in mice after various treatments. $* P<0.05$ and $* * P<0.01$ compared with the NGO-SS-HA-Gef group.

Abbreviations: NGO, nano-graphene oxide; HA, hyaluronic acid; Gef, gefitinib; HMD, hexamethylenediamine; RB, rhodamine B; GSH-OEt, glutathione reduced ethyl ester; P-EGFR, phosphorylation of epidermal growth factor receptor tyrosine; H\&E, hematoxylin and eosin; IHC, immunohistochemical.

significantly inhibit the phosphorylation of EGFR tyrosine (P-EGFR), ${ }^{43}$ which is involved in cell growth, proliferation, differentiation, and so on. The expression of P-EGFR was examined by IHC staining. The results (Figure 8F and G) revealed that among the treatment groups, the NGO-SS-HAGef groups had the lowest expression of P-EGFR. These IHC results were correlated well with the antitumor capacity observed in the abovementioned studies.

\section{Conclusion}

We developed a redox-stimuli responsive targeted delivery system based on GO nanosheets, in which HA was conjugated with the nanosheets by disulfide bonds. The resulting NGO-SS-HA showed physiological stability, admirable biocompatibility, and low cytotoxicity in vitro and in vivo. Gef could be loaded on the surface of NGO-SS-HA through $\pi-\pi$ stacking and hydrophobic interactions. However, the HA fixed 
on the surface of the nanosheets could function as a shell barrier, limiting the Gef release from NGO-SS-HA-Gef. A redoxresponsive mechanism, that is, cleavage of disulfide bonds in the presence of GSH, was used to accelerate the release of Gef from NGO-SS-HA-Gef, as evidenced by a drug release assay in vitro. Both flow cytometric analysis and confocal microscopy showed that the uptake of NGO-SS-HA by A549 cells was facilitated through CD44 receptor-mediated endocytosis and that intracellular drug release from the nanosheets could be induced in the presence of GSH. After incubation with A549 cells, NGO-SS-HA-Gef was found to significantly enhance the inhibitory activity of Gef against cell proliferation and induce cell apoptosis. Tumor-bearing mice treated with NGO-SS-HA-Gef experienced the lowest tumor growth rates among the test groups, indicating that injection of NGO-SSHA-Gef induced the strongest tumor inhibition.

\section{Acknowledgment}

This work was supported by the Ministry of Science and Technology of China (2015CB931804).

\section{Disclosure}

The authors report no conflicts of interest in this work.

\section{References}

1. Wright JR, Ung YC, Julian JA, et al. Randomized, double-blind, placebocontrolled trial of erythropoietin in non-small-cell lung cancer with disease-related anemia. J Clin Oncol. 2007;25(9):1027-1032.

2. Aupérin A, Le Péchoux C, Rolland E, et al. Meta-analysis of concomitant versus sequential radiochemotherapy in locally advanced non-small-cell lung cancer. J Clin Oncol. 2010;28(13):2181-2190.

3. Wang R, Hu H, Pan Y, et al. RET fusions define a unique molecular and clinicopathologic subtype of non-small-cell lung cancer. J Clin Oncol. 2012;30(35):4352-4359.

4. Ranson M, Davidson N, Nicolson M, et al. Randomized trial of paclitaxel plus supportive care versus supportive care for patients with advanced non-small-cell lung cancer. J Natl Cancer Inst. 2000;92(13): 1074-1080.

5. Scagliotti G, Novello S, von Pawel J, et al. Phase III study of carboplatin and paclitaxel alone or with sorafenib in advanced non-small-cell lung cancer. J Clin Oncol. 2010;28(11):1835-1842.

6. Fossella F, Pereira JR, von Pawel J, et al. Randomized, multinational, phase III study of docetaxel plus platinum combinations versus vinorelbine plus cisplatin for advanced non-small-cell lung cancer: the TAX 326 study group. J Clin Oncol. 2003;21(16):3016-3024.

7. Gervais R, Ducolone A, Breton JL, et al. Phase II randomised trial comparing docetaxel given every 3 weeks with weekly schedule as second-line therapy in patients with advanced non-small-cell lung cancer (NSCLC). Ann Oncol. 2005;16(1):90-96.

8. Scagliotti GV, Pastorino U, Vansteenkiste JF, et al. Randomized phase III study of surgery alone or surgery plus preoperative cisplatin and gemcitabine in stages IB to IIIA non-small-cell lung cancer. J Clin Oncol. 2012;30(2):172-178.

9. Liu YP, Yang CJ, Huang MS, et al. Cisplatin selects for multidrugresistant CD133+ cells in lung adenocarcinoma by activating Notch signaling. Cancer Res. 2013;73(1):406-416.
10. Sequist LV, Martins RG, Spigel D, et al. First-line gefitinib in patients with advanced non-small-cell lung cancer harboring somatic EGFR mutations. J Clin Oncol. 2008;26(15):2442-2449.

11. Mok TS, Wu YL, Thongprasert S, et al. Gefitinib or carboplatinpaclitaxel in pulmonary adenocarcinoma. $N$ Engl J Med. 2009;361(10): 947-957.

12. Zhang L, Ma S, Song X, et al; INFORM investigators. Gefitinib versus placebo as maintenance therapy in patients with locally advanced or metastatic non-small-cell lung cancer (INFORM; C-TONG 0804): a multicentre, double-blind randomised phase 3 trial. Lancet Oncol. 2012; 13(5):466-475.

13. Phillip Lee YH, Sathigari S, Jean Lin YJ, et al. Gefitinib-cyclodextrin inclusion complexes: physico-chemical characterization and dissolution studies. Drug Dev Ind Pharm. 2009;35(9):1113-1120.

14. Chitkara D, Singh S, Kumar V, et al. Micellar delivery of cyclopamine and gefitinib for treating pancreatic cancer. Mol Pharm. 2012;9(8):2350-2357.

15. Godugu C, Doddapaneni R, Patel AR, Singh R, Mercer R, Singh M. Novel Gefitinib Formulation with Improved Oral Bioavailability in Treatment of A431 Skin Carcinoma. Pharm Res. 2016;33(1):137-154.

16. Eda G, Chhowalla M. Chemically derived graphene oxide: towards large-area thin-film electronics and optoelectronics. Adv Mater. 2010; 22(22):2392-2415.

17. Compton OC, Jain B, Dikin DA, Abouimrane A, Amine K, Nguyen ST. Chemically active reduced graphene oxide with tunable $\mathrm{C} / \mathrm{O}$ ratios. $A C S$ Nano. 2011;5(6):4380-4391.

18. Sheng ZH, Shao L, Chen JJ, Bao WJ, Wang FB, Xia XH. Catalyst-free synthesis of nitrogen-doped graphene via thermal annealing graphite oxide with melamine and its excellent electrocatalysis. ACS Nano. 2011; 5(6):4350-4358.

19. Ma X, Tao H, Yang K, et al. A functionalized graphene oxide-iron oxide nanocomposite for magnetically targeted drug delivery, photothermal therapy, and magnetic resonance imaging. Nano Res. 2012;5(3):199-212.

20. Wang Z, Dong Y, Li H, et al. Enhancing lithium-sulphur battery performance by strongly binding the discharge products on aminofunctionalized reduced graphene oxide. Nat Commun. 2014;5:5002.

21. Sun X, Liu Z, Welsher K, et al. Nano-graphene oxide for cellular imaging and drug delivery. Nano Res. 2008;1(3):203-212.

22. Song E, Han W, Li C, et al. Hyaluronic acid-decorated graphene oxide nanohybrids as nanocarriers for targeted and $\mathrm{pH}$-responsive anticancer drug delivery. ACS Appl Mater Interfaces. 2014;6(15):11882-11890.

23. Surace C, Arpicco S, Dufä̈-Wojcicki A, et al. Lipoplexes targeting the CD44 hyaluronic acid receptor for efficient transfection of breast cancer cells. Mol Pharm. 2009;6(4):1062-1073.

24. Huang Y, Yao X, Zhang R, et al. Cationic conjugated polymer/fluoresceinamine-hyaluronan complex for sensitive fluorescence detection of CD44 and tumor-targeted cell imaging. ACS Appl Mater Interfaces. 2014;6(21):19144-19153.

25. Dreaden EC, Morton SW, Shopsowitz KE, et al. Bimodal tumortargeting from microenvironment responsive hyaluronan layer-by-layer (LbL) nanoparticles. ACS Nano. 2014;8(8):8374-8382.

26. Wen H, Dong C, Dong H, et al. Engineered redox-responsive PEG detachment mechanism in PEGylated nano-graphene oxide for intracellular drug delivery. Small. 2012;8(5):760-769.

27. Schafer FQ, Buettner GR. Redox environment of the cell as viewed through the redox state of the glutathione disulfide/glutathione couple. Free Radic Biol Med. 2001;30(11):1191-1212.

28. Zhao Q, Geng H, Wang Y, et al. Hyaluronic acid oligosaccharide modified redox-responsive mesoporous silica nanoparticles for targeted drug delivery. ACS Appl Mater Interfaces. 2014;6(22):20290-20299.

29. Ma YC, Wang JX, Tao W, et al. Redox-Responsive PolyphosphoesterBased Micellar Nanomedicines for Overriding Chemoresistance in Breast Cancer Cells. ACS Appl Mater Interfaces. 2015;7(47):26315-26325.

30. Pan YJ, Chen YY, Wang DR, et al. Redox/pH dual stimuli-responsive biodegradable nanohydrogels with varying responses to dithiothreitol and glutathione for controlled drug release. Biomaterials. 2012; 33(27):6570-6579. 
31. Cheng R, Feng F, Meng F, Deng C, Feijen J, Zhong Z. Glutathioneresponsive nano-vehicles as a promising platform for targeted intracellular drug and gene delivery. J Control Release. 2011;152(1):2-12.

32. Marcano DC, Kosynkin DV, Berlin JM, et al. Improved synthesis of graphene oxide. ACS Nano. 2010;4(8):4806-4814.

33. Liu Z, Robinson JT, Sun X, Dai H. PEGylated nanographene oxide for delivery of water-insoluble cancer drugs. J Am Chem Soc. 2008; 130(33):10876-10877.

34. Wang J, Cheng M, Zhang Z, Guo L, Liu Q, Jiang G. An antibodygraphene oxide nanoribbon conjugate as a surface enhanced laser desorption/ionization probe with high sensitivity and selectivity. Chem Commun. 2015;51(22):4619-4622.

35. Huang $\mathrm{P}, \mathrm{Xu} \mathrm{C}$, Lin J, et al. Folic Acid-conjugated Graphene Oxide loaded with Photosensitizers for Targeting Photodynamic Therapy. Theranostics. 2011;1:240-250.

36. Ren W, Fang Y, Wang E. A binary functional substrate for enrichment and ultrasensitive SERS spectroscopic detection of folic acid using graphene oxide/Ag nanoparticle hybrids. ACS Nano. 2011;5(8): 6425-6433.

37. Zhou T, Zhou X, Xing D. Controlled release of doxorubicin from graphene oxide based charge-reversal nanocarrier. Biomaterials. 2014;35(13):4185-4194.
38. Duch MC, Budinger GR, Liang YT, et al. Minimizing oxidation and stable nanoscale dispersion improves the biocompatibility of graphene in the lung. Nano Lett. 2011;11(12):5201-5207.

39. Zhang L, Xia J, Zhao Q, Liu L, Zhang Z. Functional graphene oxide as a nanocarrier for controlled loading and targeted delivery of mixed anticancer drugs. Small. 2010;6(4):537-544.

40. Dong H, Gao W, Yan F, Ji H, Ju H. Fluorescence resonance energy transfer between quantum dots and graphene oxide for sensing biomolecules. Anal Chem. 2010;82(13):5511-5517.

41. Shen L, Li Z, Shen S, et al. The synergistic effect of EGFR tyrosine kinase inhibitor gefitinib in combination with aromatase inhibitor anastrozole in non-small cell lung cancer cell lines. Lung Cancer. 2012;78(3): 193-200.

42. Rho JK, Choi YJ, Ryoo BY, et al. p53 enhances gefitinib-induced growth inhibition and apoptosis by regulation of Fas in non-small cell lung cancer. Cancer Res. 2007;67(3):1163-1169.

43. Sharma J, Lv H, Gallo JM. Intratumoral modeling of gefitinib pharmacokinetics and pharmacodynamics in an orthotopic mouse model of glioblastoma. Cancer Res. 2013;73(16):5242-5252.
International Journal of Nanomedicine

\section{Publish your work in this journal}

The International Journal of Nanomedicine is an international, peerreviewed journal focusing on the application of nanotechnology in diagnostics, therapeutics, and drug delivery systems throughout the biomedical field. This journal is indexed on PubMed Central, MedLine, CAS, SciSearch ${ }^{\circledR}$, Current Contents ${ }^{\circledR} /$ Clinical Medicine,

\section{Dovepress}

Journal Citation Reports/Science Edition, EMBase, Scopus and the Elsevier Bibliographic databases. The manuscript management system is completely online and includes a very quick and fair peer-review system, which is all easy to use. Visit http://www.dovepress.com/ testimonials.php to read real quotes from published authors. 